\title{
UNDERWATER SOURCE LOCALIZATION WITH A GENERALIZED LIKELIHOOD RATIO PROCESSOR,
}

\author{
A Thesis Presented to \\ The Faculty of the Russ College of Engineering and Technology \\ Ohio University
}

\author{
In Partial Fulfillment \\ of the Requirements for the Degree \\ Master of Science
}

by

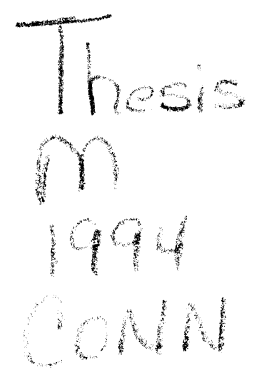

June, 1994

\section{OHIO UNWERSITY \\ LUERARY}




\section{ACKNOWLEDGMENTS}

I would like to take the opportunity to thank several people without whose help this work would not have been possible.

I would like to begin by thanking Dr. John Tague. Your help in and out of the classroom has been instrumental to my education. Also, making the whole experience enjoyable has been Star Trek, sports talks, and pizza! We also are the only ones at O.U. that recognize the true power house in Big Ten football is Penn State. Luv ya Blue!!!!!!!!!

Much appreciation is extended to the various professors in the EE department that have made my graduate courses at times frustrating but actually still enjoyable.

I, Genghis, would like to thank Mr. Tim Bambeck for providing me with the opportunity to teach. I enjoyed it more then I thought possible. You also let me be your student and taught me a great deal about engineering.

Much appreciation is extended to Dr. H. Bryan Riley who has extended a great deal of assistance in regards to this thesis. Your time and patience is deeply appreciated.

To the ladies that run the department Janelle, Denise, Jo Anne, and Suzanne, I would like to thank you for all your help. You have provided me not only with your technical assistance but also many humorous times in the EE department.

Personal thanks go to Mr. Rick Cooper and Mr. Bill Cooper the best neighbors in the world. Here's to cable TV, cookouts, playing in the rain, snowball fights, dinners, softball, Disney cartoons, and red roses on Valentine's day. Thank you!

To Denise Kanya who has put up with my ups and downs of graduate school. Your assistance in this work can not begin to be measured. Simply put, you have been a great roommate and a best friend. Thanks Poopsie! 
I especially would like to thank my mother, father, and entire family. You have always believed in me, even when I didn't believe in myself. My greatest pride will always come in being a member of such a fantastic family.

Finally, I would like to thank my better half (I can't believe I put that in print) David Bartholomew. The best thing I received at O.U. was you!! 


\section{TABLE OF CONTENTS}

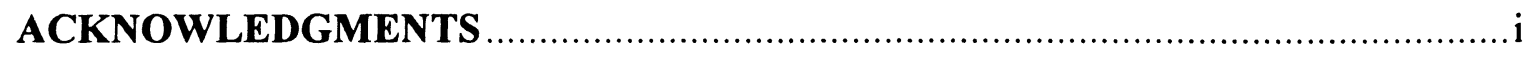

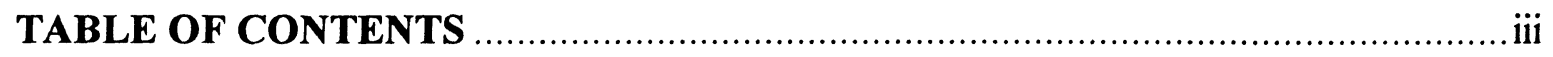

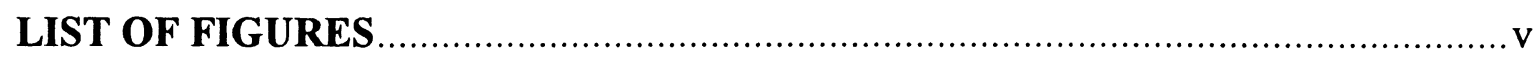

\section{CHAPTER}

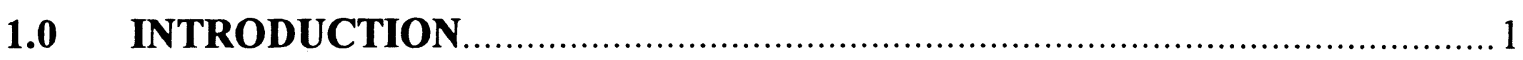

1.1 Statement Of The Problem ................................................................ 1

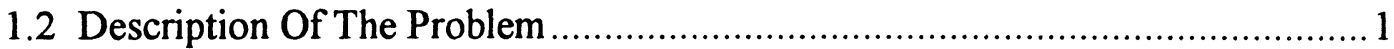

1.3 Motivation And Objective ................................................................ 3

1.4 Organization Of The Thesis ............................................................ 4

2.0 ARRAY SIGNAL PROCESSING .................................................... 5

2.1 Introduction ...................................................................................... 5

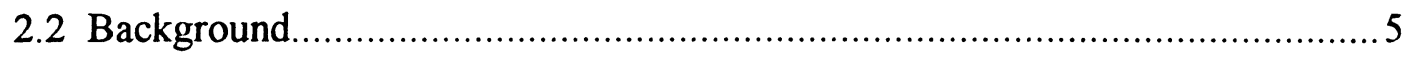

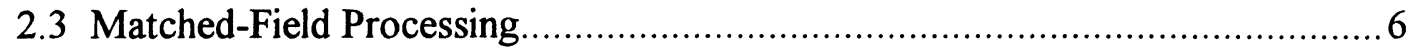

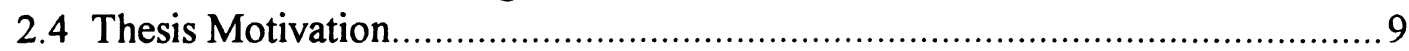

2.5 Bartlett Estimator ...........................................................................

2.6 The Maximum Likelihood Estimator........................................................ 11

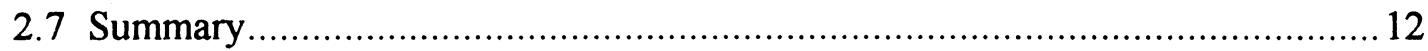

3.0 PROBLEM FORMULATION ......................................................... 13

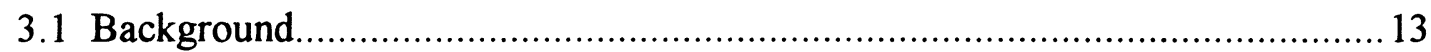

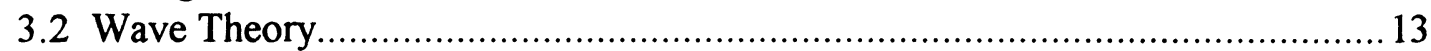

3.2.1 The Parabolic Equation Method .................................................. 14

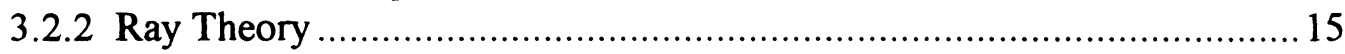

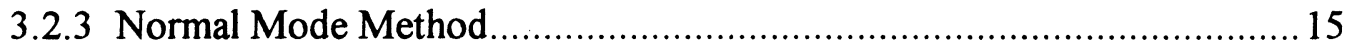

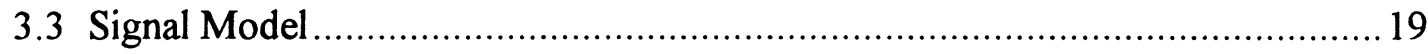

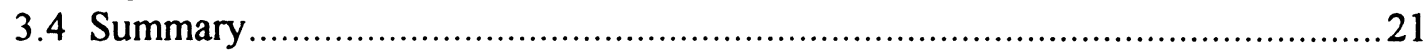

4.0 THE GENERALIZED LIKELIHOOD RATIO PROCESSOR .....................22

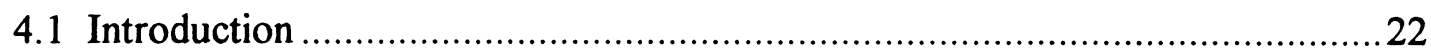

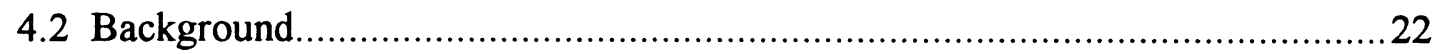

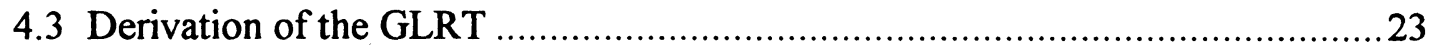

4.3.1 Optimum Detector: Known source location, source power, and noise covariance matrix......................................................... 24

4.3.2 Unknown source power and noise covariance matrix ..........................28

4.3.3 Maximizing the GLRT denominator ............................................ 28

4.3.4 Maximizing the GLRT numerator ............................................. 31

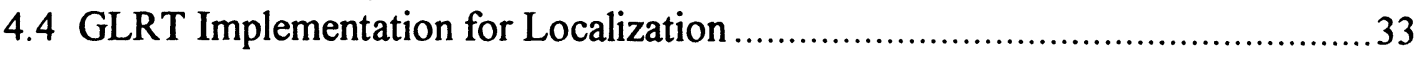




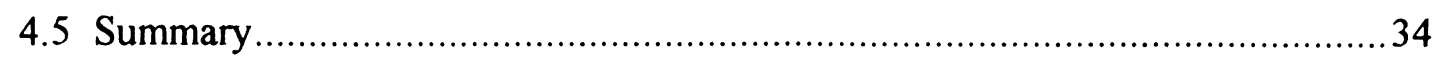

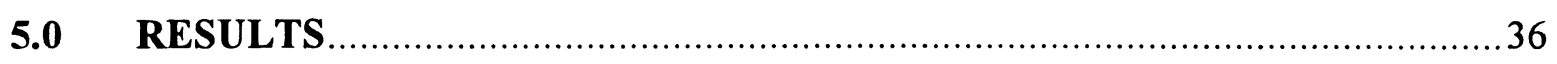

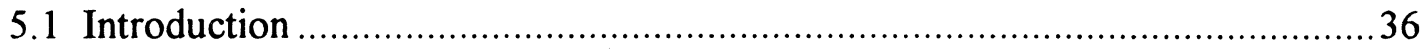

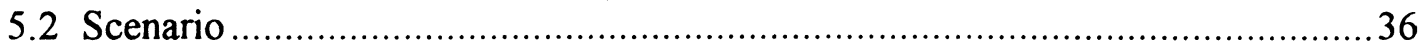

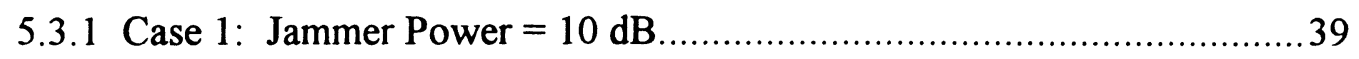

5.3.2 Case 2: Jammer Power $=0 \mathrm{~dB}$............................................... 43

5.3.3 Case 3: Jammer Power $=-10 \mathrm{~dB}$................................................. 47

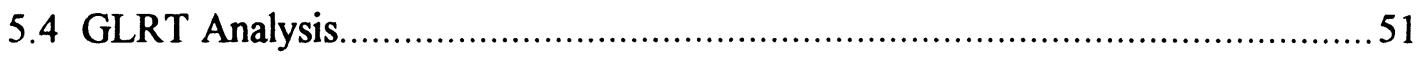

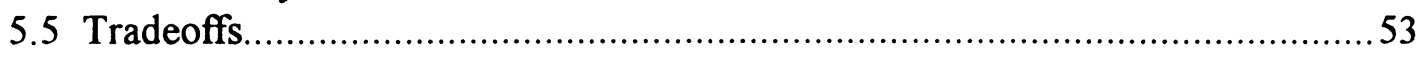

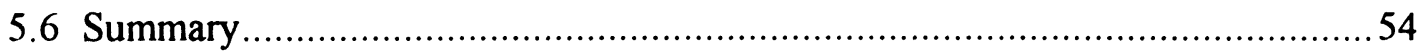

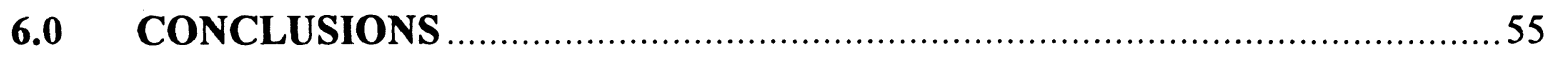

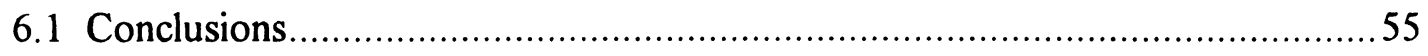

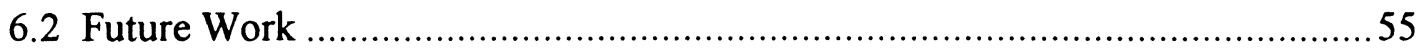

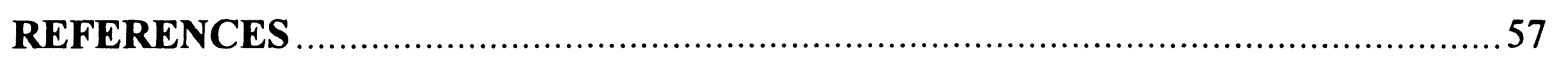

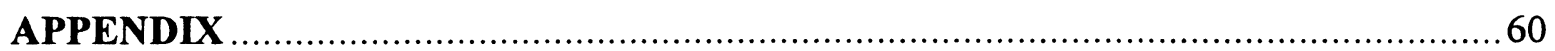




\section{LIST OF FIGURES}

Figure 1.1 Source Description..........................................................................

Figure 2.1 Layered Waveguide .................................................................. 8

Figure 5.1 Layered Waveguide For Simulations ................................................... 37

Figure 5.2 MFP Case 1: Bartlett Ambiguity Surface .................................................... 40

Figure 5.3 MFP Case 1: MLM Ambiguity Surface ............................................. 41

Figure 5.4 MFP Case 1: GLRT Ambiguity Surface ............................................ 42

Figure 5.5 MFP Case 2: Bartlett Ambiguity Surface .............................................. 44

Figure 5.6 MFP Case 2: MLM Ambiguity Surface .......................................... 45

Figure 5.7 MFP Case 2: GLRT Ambiguity Surface ........................................... 46

Figure 5.8 MFP Case 3: Bartlett Ambiguity Surface ................................................ 48

Figure 5.9 MFP Case 3: MLM Ambiguity Surface ................................................. 49

Figure 5.10 MFP Case 3: GLRT Ambiguity Surface ..................................................50 


\section{CHAPTER ONE}

\section{INTRODUCTION}

\subsection{Statement Of The Problem}

This thesis studies underwater source detection and localization using MatchedField Processing, an underwater source localization technique that incorporates environmental information into the signal processing algorithm.

In this thesis a generalized likelihood ratio test (GLRT) is derived and applied to a prototype underwater signal processing problem. The GLRT improves on current signal processing estimators currently used in MFP applications. The estimators most often implemented can not effectively suppress strong directional interference. This leads to ambiguous results. Our research provides the means to accurately localize and detect a source by removing the strong directional interference. This is accomplished by incorporating an interference model into the signal processing algorithm.

\subsection{Description Of The Problem}

The Matched-Field Processing procedure is illustrated in Figure 1.1. An acoustic source generating a narrowband signal is located at an unknown range and depth in an acoustic waveguide. The shallow water environment that the signal propagates through acts as a waveguide. If plane wave propagation is assumed then the performance of the

processor would be greatly degraded; therefore, the environment must be modeled using more complex procedures. Complicating the problem is interference present in the environment, which obscures the source signal. A vertical hydrophone array collects 
complex phone signals and sends the data to the processor. The problem is to detect and localize the source.

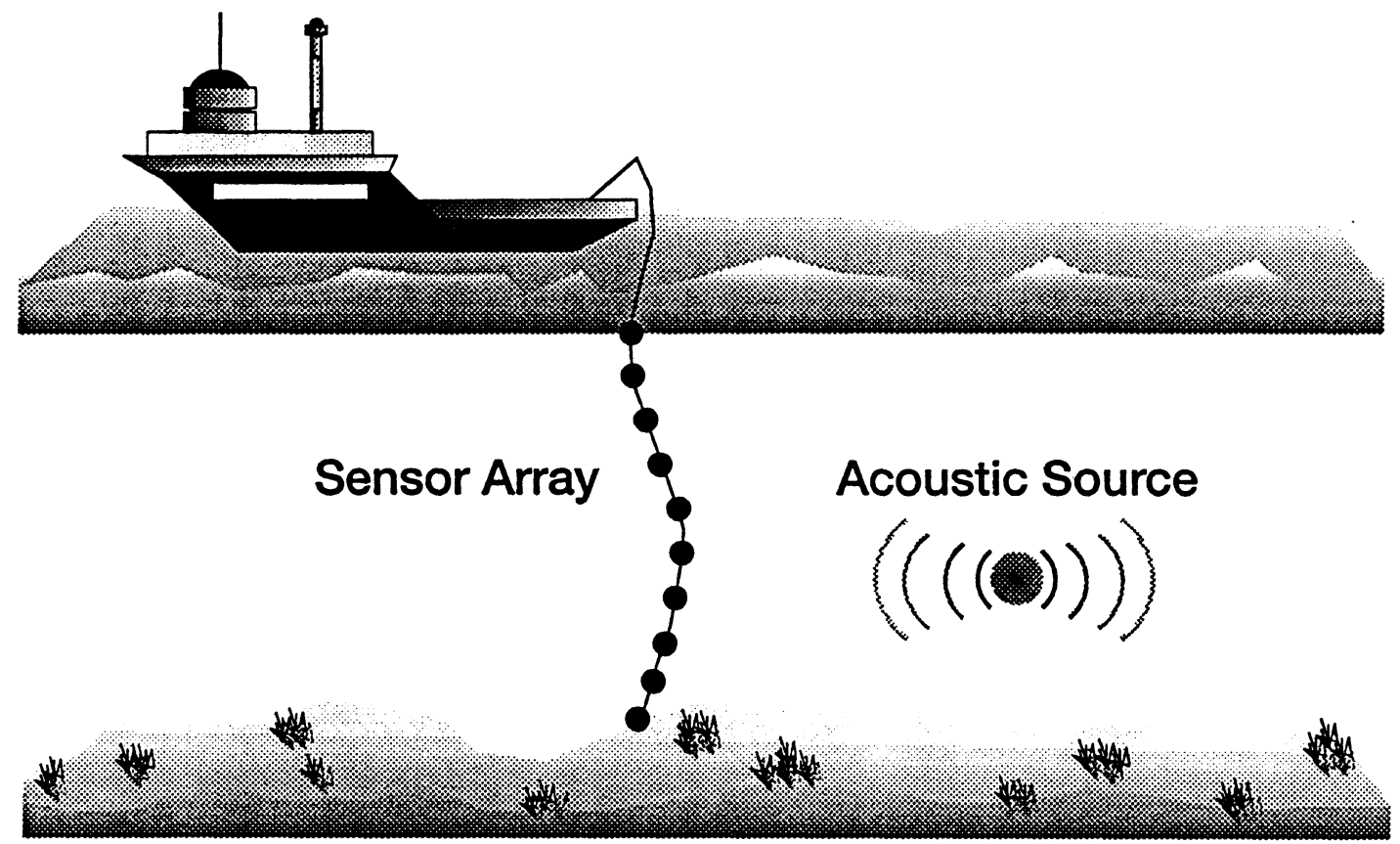

Figure 1.1 Source Description. 
This is accomplished by applying Matched-Field techniques using a "reduced-rank" array processor. Reduced rank processing can be applied when the number of modes are less then the number of sensors in the sensor array. The modes are defined as the number of jammers present, which are clearly defined further in the thesis.

\subsection{Motivation and Objective}

The basis for this research originates in the limitations of conventional estimators. Conventional estimators, such as the Bartlett and Maximum Likelihood Method (MLM), are unable to effectively remove strong directional interference. This hinders the processor and provides ambiguous results regarding the "true" location of the source. Furthermore, these estimators are not good detectors.

The parameter estimator that we derive in this thesis is the generalized likelihood ratio test (GLRT) $[4,5,6]$. We will show that this processor is capable of suppressing sources of strong directional interference. Another factor taken into account is to accurately model the signal fields. Usually, in array processor design and implementation, it is assumed that energy propagates in plane waves. However, in shallow water environments, if plane wave propagation is assumed, performance is severely degraded. Thus, accurate models for the signal and jammer fields are essential. Another consideration made throughout this work is that the strength of the noise components is comparable to the source. The final objective of our research is that through the implementation of the GLRT processor, the desired source would be detected and localized. 


\subsection{Organization Of The Thesis}

The thesis consists of six chapters. The first chapter contains a brief introduction of the thesis as well as the goals of our research.

The second chapter gives a thorough explanation of Matched-Field Processing and its role in this research. Chapter Two also discusses conventional source position estimators. The goal of this discussion is to enable the reader to see the motivation behind the development of a new parameter estimator.

Chapter Three discusses the formulation of the problem and the signal model. This thesis attempts to present a working environment that is similar to a "real world" scenario. In chapter three a discussion on wave theory and various modeling methods is included.

In Chapter Four we will derive the generalized likelihood ratio processor. The derivation covers the mathematical and theoretical process of the GLRT. The final result is a ratio that will allow us to detect and localize the source and jammers present. The plots will have distinct peaks to indicate the source signal. Chapter Four will explain why and how these results are achievable.

Chapter Five is the results section of the thesis. Also, Chapter Five presents an analysis of the GLRT useful to understand the results described in this chapter.

The final chapter contains the conclusions that were reached at the end of the research. Suggestions for future research dealing with this subject are also found in this chapter. 


\section{CHAPTER TWO}

\section{ARRAY SIGNAL PROCESSING}

\subsection{Introduction}

The topic discussed in Chapter Two is array signal processing. Therefore, the background discussion includes an overview of spatial processing and its applications. The goals of the remaining section of the chapter are two fold. The first section is to give the reader a basic understanding of the theory and procedure of Matched-Field Processing. Finally, a discussion on estimators currently used in Matched Field techniques is included. This is to provide the reader with an understanding behind the motivation for a new processor design.

\subsection{Background}

Matched-Field Processing (MFP) utilizes beamforming, the central array signal processing technique. Beamforming is the signal processing method of spatially filtering multisensor data to form beams for the reception of energy [18]. The wide range of applications include antenna theory, ultrasound, biomedical engineering, signal processing, electromagnetics, and array signal processing.

A beamformer is a spatial filtering system which is designed to separate signals that originate from different bearings or spatial locations. The ambient noise and signals arriving from various directions are measured with a sensor array. The acoustical energy is converted into electrical energy by transducers. The data from the array that is used for processing are measurement vectors or "snapshots". Theoretically, the directions from which the signals propagate can be extracted from the data. The output of the sensor 
array is modified to suppress background noise along with undesired signals while enhancing the desired signal reception. An assumption made using this process is that the wavefields are homogeneous; that is, a superposition of uncorrelated waves.

The underlying idea is that if a propagating signal is present in the array's field of view, the sensor outputs, delayed by appropriate amounts and added together, reinforce the signal with respect to noise or waves propagating in different directions [3]. The delays are related to the length of time it takes the signal to propagate between the sensors. The conventional delay-and-sum beamformer is represented as

$$
z(t)=\sum_{m=0}^{M-1} a_{m} y_{m}\left(t-\Delta_{m}\right)
$$

where $\left\{a_{m}\right\}$ are the amplitude weight to the output of each sensor. This is often referred to as the array's shading or taper. $\left\{y_{m}\right\}$ represents the measurement of the waveform at the $m^{\text {th }}$ sensor. $M$ is the number of sensors in the array and $\Delta_{m}$ represents the delay. The amplitude weights are generally chosen such that the beam can be steered in a particular direction. The frequency domain representation of the sum-and-delay beamformer is given by

$$
\mathbf{Y}(w, \theta)=\sum_{n=0}^{N} y_{m}(w) a_{m} e^{-j w \tau_{n}}=\mathbf{A}^{\mathbf{H}} \mathbf{X}
$$

\subsection{Matched-Field Processing}

Matched-Field Processing (MFP) is a relatively new application of array signal processing techniques. It was specifically designed for shallow water environments in underwater signal processing. MFP is a beamformer technique that incorporates 
environmental information into the signal processing algorithm. The objective is to estimate the location of a point acoustic source by "matching" the source field to the field we compute in our search for the source. The ocean environment is only several hundred meters deep; therefore, it is referred to as "shallow water". The shallow water environment will induce waveguide acoustic propagation conditions.

MFP can be explained through the use of Figure 2.1. The source location is denoted by the coordinates $r_{o}$ and $z_{o}$ for range and depth, respectively. It emits a narrowband signal which propagates through the shallow water environment. Acting to obscure the source are various noise components inherent to the ocean. The measurement array, which can lie in a vertical or horizontal configuration, receives the signal. The measurement array is composed of $n$ evenly spaced sensors or hydrophones. The data that is collected is then sent to the processor. The processor that is implemented must then estimate the source location. This is accomplished through the use of MFP.

The principle of matched field processing is described as follows. We must use non trivial acoustic propagation models to characterize the shallow water channel. Plane wave propagation can not be assumed due to the waveguide conditions, to do so would degrade the performance of the processor. The propagation model solves the wave equation for a given set of boundary conditions which describe the underlying propagational channel structure. Several methods can be used to model the propagating fields and are discussed further in Chapter Three. A complex acoustic field is simulated as a function of the source location. This is referred to as the replica field and denoted by the 


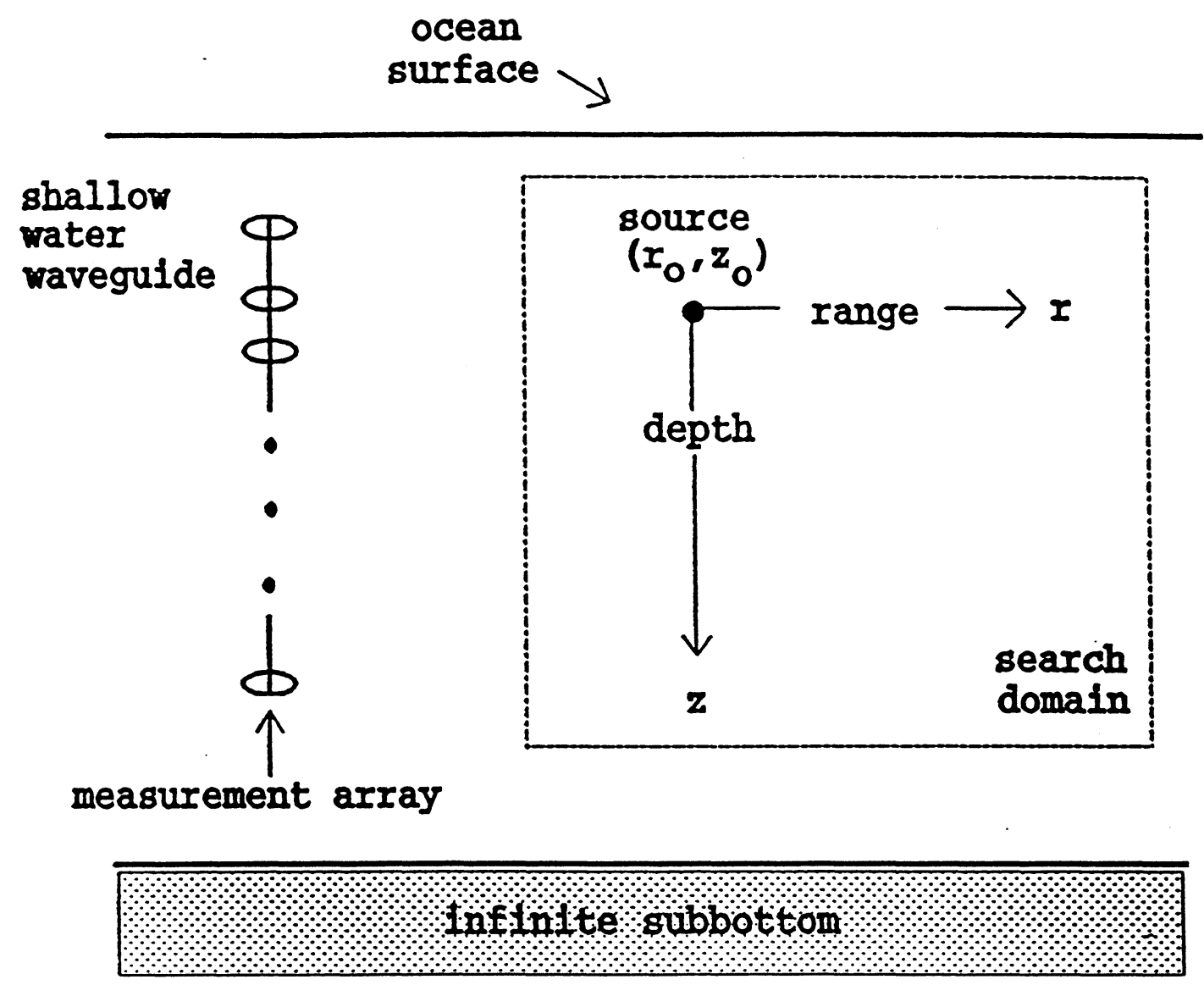

Figure 2.1 Layered Waveguide. Source in a water layer over sediment. The position of the source varies as $\left(r_{o}, z_{o}\right)$, and the position of the receiver is fixed. 
dotted lines in Figure 2.1. It models the recorded complex pressure field recorded at the $n^{\text {th }}$ element of the vertical hydrophone array due to the source located at an hypothesized range and depth [12]. We compute the replica field for an extensive number of assumed source range and depth locations. The replica fields, are sent one per hypothesized source location, to the array processor. Thus, environmental information is incorporated into the signal processing algorithm. The processor compares the replica field to actual ocean measurements using a variety of estimation procedures. Estimates of point source locations are determined by peaks in the output.

\subsection{Thesis Motivation}

The next two sections describe the processors currently used for MFP. Their, limitations provide the motivation for a new processor. For example, the processors that are discussed (Bartlett and MLM) are not specifically designed for detection. Also, if the directional interference present in the environment (jammer(s)) is strong, it will effectively "blot out" the estimated source peak. Therefore, our goal is to design a processor that can detect and localize the source in the presence of strong directional interference. The strength of the source, jammers, and white noise is unknown, as well as the locations of the source and jammers.

\subsection{The Bartlett Estimator}

The Bartlett estimator is the most common power estimator used in MFP. It works by collecting a set of $\mathrm{K}$ array measurements, $x_{1}, \ldots, x_{K}$. Next, the array covariance matrix is estimated 


$$
\hat{R}=\frac{1}{K} \sum_{k=1}^{K} x_{k} x_{k}^{H}
$$

The array measurements that are collected to estimate $\mathrm{R}$ include information about the ambient noise, jammers, and source. We can say that the estimated power that originates from location $(r, z)$ is given by

$$
\hat{P}_{B A R T}(\hat{r}, \hat{z})=e^{H}(\hat{r}, \hat{z}) \hat{R} e(\hat{r}, \hat{z})
$$

$\underline{e}(r, z)$ is the steering vector or in matched field terms, the replica field vector. $r$ and $z$ denote the range and depth coordinates respectively. $\underline{e}(r, z)$ is obtained by solving the wave equation for the pressure field measured at the array assuming a hypothetical point acoustic source located at $(r, z)$. The best results are achieved when a large number of trial solutions are constructed. This is achieved by steering the "beam" through a selected search sector. An estimate of the source location is determined by the maximum value of the power as $(r, z)$ varies throughout the search region. A three dimensional plot of the result is known as an ambiguity surface. The plot reflects both range and depth coordinates. Peaks in $\hat{P}_{B A R T}(r, z)$ correspond to estimates of point source locations. The Bartlett estimator is known for its computational efficiency and its ease of implementation. However, the high sidelobes in the output lead to ambiguous results when estimating the source location. In addition, the Bartlett estimator is not specifically designed for detection purposes. 


\subsection{The Maximum Likelihood Method}

The Maximum Likelihood Method (MLM) was proposed by Capon for use in processing seismic data. The motivation behind its development was to solve the sidelobe problems associated with the Bartlett estimator. The derivation of the MLM estimator is accomplished in the following manner. We want to minimize the effects that result in the high side lobes. This is accomplished by finding a weight vector $w$ which will minimize the variance of the processor response to the total field, subject to a unit gain constraint in the look direction. Therefore, the problem is formulated as follows:

$$
\min _{w} w R w \quad \text { subject to } \quad w^{H} e(r, z)=1 .
$$

The solution is given by the weight vector

$$
w_{M L M}=\frac{R^{-1} e(r, z)}{e(r, z)^{H} R^{-1} e(r, z)} .
$$

The weights of this beamformer are such that the spatial spectrum is a maximum likelihood estimate of the power being received as a function of wavenumber (angle) if the noise-only covariance matrix is known [11]. Substituting equation (2.6) into equation (2.4) yields

$$
P_{M L M}(r, z)=\frac{1}{e^{H}(r, z) R^{-1} e(r, z)} .
$$

If $R$ is unknown, then the ML estimate given in equation (2.7) is used. 
The MLM method provides greater sidelobe reduction and superior resolving power as compared to the Bartlett method. The tradeoff between the two processors is speed. The MLM processor is slower due to the inversion of the covariance matrix $R$. If the matrix is large, as often is the case in practical situations, the process is considerably slower. However, the peaks in the output are very distinct thereby giving good estimates of the source location. The MLM is a power estimator and like the Bartlett estimator is not specifically designed for detection.

\subsection{Summary}

The purpose of Chapter Two was to explain a new procedure applied to shallow water environments. In the context of MFP a processor must be implemented to process the data and estimate the source location. The question is, which processor gives the best estimates? The conventional estimators were discussed and their limitations listed. The motivation for our work, to develop a new processor, lies in the limitations of the current estimators. The Generalized Likelihood Ratio Test (GLRT) is derived and discussed in Chapter Four. The modeling techniques that were summarized in this chapter are discussed in detail in Chapter Three. 


\section{CHAPTER THREE \\ PROBLEM FORMULATION}

\subsection{Background}

This chapter discusses the formulation of the problem. This entails modeling the measurements, as well as the signal, jammer, and replica fields. Section 3.2 discusses wave theory. The purpose of this section is to describe various methods in which the fields can be modeled. This section should leave the reader with a clear understanding as to why the normal mode model was chosen. The final section of this chapter describes how the problem was formulated and why the measurements were modeled in the manner chosen.

\subsection{Wave Theory}

This section describes various methods available to model propagating fields. In underwater acoustics, the medium and boundaries can vary spatially (in range, depth, and azimuth) as well as in time. Acoustic propagation is described by a pressure disturbance or field traveling through the ocean. It satisfies the wave (time-dependent) or Helmholtz (time-independent) equations where sound speed plays the same role as the index of refraction in optics [1]. The methods briefly discussed are well known methods and are used in various applications in signal processing, electromagnetics and antenna theory.

The time-independent Helmholtz equation is obtained from the general inhomogeneous wave equation which describes sound propagation in water. The Helmholtz equation for a point source with unity pressure amplitude in cylindrical coordinates is 


$$
\left\{\frac{1}{r} \frac{\partial}{\partial r}\left(r \frac{\partial}{\partial r}\right)+\frac{\partial^{2}}{\partial z^{2}}+\left[\frac{\omega}{c(z)}\right]^{2}\right\} \mathbf{p}=-\frac{2}{r} \delta(r) \delta\left(z-z_{o}\right) e^{j \omega t}
$$

where $\omega$ is the angular frequency and $c(z)$ is the speed of sound in water as a function of depth. The solution to equation (3.1) describes the acoustic far field in the ocean, produced by a single frequency continuous wave point source at $\left(r=r_{o}, z=z_{o}\right)$. We next describe how to solve (3.1) in shallow water environments.

\subsubsection{The Parabolic Equation Method}

The PE method solves a parabolic equation which approximates the Helmholtz equation assuming that the received field results from only a restricted cone of shallow source launch angles [2]. This is a reasonable long and medium range propagation assumption. The advantages to using the PE model are the numerous numerical methods available to solve the parabolic equations. The overall research of ocean fronts, bathymetry, and geoacoustics of the seabed has refined several other PE methods for modeling propagation at low frequency [19] and [20]. This, combined with the improvement of high speed processing units, has led to more time efficient and accurate results. Another key advantage to the PE method is its capability to handle energy loss due to absorption and transmission in the bottom.

A disadvantage to using the PE method is its limitation to nearly radial propagation. Radial propagation occurs when large downward sloping bottoms appear near the source [2]. Also, a starting field is required to initialize the solution when using the PE. Finally, PE models do not generally incorporate back scattering of energy and tend to have computational difficulties with increasing environmental complexities and 
frequency. The PE models tend to have phase errors which will accumulate with increasing range. This will greatly affect MFP results.

\subsubsection{Ray Theory}

A ray is defined as a line everywhere perpendicular to the surfaces of constant phase. The medium is effectively divided into small layers, which results in ray refraction according to Snell's law. When a wave is restricted to a limited solid angle (i.e. a beam of sound from a highly directional source), descriptions in terms of rays is much easier than in terms of wave fronts [2]. The advantage of ray theory is that solutions are rapidly computed, highly intuitive, and are easily visualized. The disadvantage to using ray theory is that low frequency behavior is not taken into account. Thus, since MFP is generally used in low frequency applications, ray theory is rarely used in MFP problems.

\subsubsection{Normal Mode Method}

Normal mode theory is made to order for depth-dependent profiles. In this research, the Normal Mode (NM) Method was used to generate the replica, signal, and jammer fields. This method is the most commonly used method in MFP. The NM method is derived from the Helmholtz equation by assuming a separable coordinate system that is appropriate to the given problem. The solution is then expressed as a finite sum of depthdependent eigenfunctions or resonant modes which are analogous to the vibration modes of a string. The modes satisfy local boundary conditions which in turn are determined by the bottom structure, surface, and sound velocity profile (SVP). NM theory has been used in underwater acoustics to study mixed layers, Arctic waters, and other ducted propagation examples. 
The general theory for many layered problems was developed by Tolstoy and Clay [21]. The theoretical background to obtain the normal mode solutions due to a point source in cylindrical coordinates was examined carefully through this research. The Helmholtz equation for a point source of pressure amplitude $P(1)=1$ is given in equation (3.1). It is assumed that acoustic energy is contained within a sound channel; therefore, it is possible to represent the solution as a linear combination of eigenfunctions:

$$
\mathbf{p}(r, z, t)=e^{j \omega x} \sum_{n} \mathbf{R}_{n}(r) Z_{n}(z)
$$

where the $\mathbf{R}_{n}(r)$ describes the cylindrical spreading, and $Z_{n}(z)$ satisfies some Helmholtz equation [2]. We postulate that $Z_{n}(z)$ must satisfy the equation

$$
\frac{d^{2} Z_{n}}{d z^{2}}+\left[\frac{\omega^{2}}{c^{2}(z)}-K_{n}^{2}\right] Z_{n}=0
$$

where $K_{n}$ is a constant. It can be shown that subject to certain "reasonable" boundary conditions, $Z_{n}(z)$ forms an orthogonal set of functions normalized so that

$$
\int Z_{n}(z) Z_{m}(z) d z=\delta_{n m}
$$

where $\delta_{n m}=0$ unless $\mathrm{n}=\mathrm{m}$, for which $\delta_{n n}=1$ [2]. The above equation holds as long as the separation constants $K_{n}$ and $K_{m}$ are not equal. Substitution of (3.2) and (3.3) into (3.1) yields

$$
\sum_{n}\left[Z_{n} \frac{1}{r} \frac{d}{d r}\left(r \frac{d \mathbf{R}_{n}}{d r}\right)+\mathbf{R}_{n} K_{n}^{2} Z_{n}\right]=-\frac{2}{r} \delta(r) \delta\left(z-z_{o}\right)
$$


When you multiply the equation by $Z_{m}(z)$, integrate over the depth, and simplify it with the orthonormality condition, this yields

$$
\frac{1}{r} \frac{d}{d r}\left(r \frac{d \mathbf{R}_{n}}{d r}\right)+K_{n}^{2} \mathbf{R}_{n}=-\frac{2}{r} \delta(r) Z_{n}\left(z_{o}\right) .
$$

The solution to this inhomogeneous Helmholtz equation is $\mathbf{R}_{n}(r)=-j \pi Z_{n}\left(z_{o}\right) H_{o}^{(2)}\left(K_{n} r\right)$ where $H_{o}^{(2)}=J_{o}-j Y_{o}$ is a Hankel function [2]. It can be shown that for $r>>1$,

$$
\mathbf{p}(r, z, t)=-j \sum_{n} \sqrt{\frac{2 \pi}{K_{n} r}} Z_{n}\left(z_{o}\right) Z_{n}(z) e^{j\left(\omega t-K_{n} r+\pi / 4\right)}
$$

where the Hankel function has been replaced by its asymptotic form [2].

Under certain conditions, analytic solutions for $Z_{n}(z)$ and the horizontal wavenumbers $\left\{K_{n}\right\}$ can be obtained. Suppose that the shallow water channel has a rigid bottom and a uniform sound velocity profile (SVP) [2]. Furthermore, assume that the isospeed channel is bounded at $z=0$ by a pressure release surface and is supported at $z=H$ by a perfectly rigid bottom, where $H$ is the depth of the water. The boundary conditions are that on the surface $p=0$ and on the bottom $\partial p / \partial z=0$. Since in normal mode theory the variables separate, these boundary conditions can be applied to the eigenfunction $Z_{n}(z)$ as follows:

$$
Z_{n}(0)=0 \quad \text { and } \quad\left(\frac{\partial Z_{n}}{\partial z}\right)_{z=H}=0
$$

Since we are operating in an isospeed channel, the velocity equals the speed of sound. Therefore, $c(z)=c_{o}=1500 \mathrm{~m} / \mathrm{sec}$, and using the boundary conditions stated above, we determine that the normalized eigenfunctions are 


$$
Z_{n}(z)=\sqrt{\frac{2}{H}} \sin k_{z n} z,
$$

where $0 \leq z \leq H$. The eigenvalues or vertical wave numbers are

$$
k_{z n}=\left(n-\frac{1}{2}\right) \frac{\pi}{H}
$$

for $n \geq 1$.

We determine the values for $K_{n}$, the horizontal wave numbers from the eigenvalues:

$$
K_{n}=\sqrt{\left(\frac{\omega}{c_{o}}\right)^{2}-k_{z n}}
$$

All values for $K_{n}$ must be real, because imaginary results in equation (3.11) produce modes that will decay rapidly with increasing range. This is due to the waveguide condition that exists in a shallow water environment that produces a constant cross section. To determine the number of normal modes that propagate, we use the following formula to determine the upper bound:

$$
M \leq \frac{H}{\pi} \frac{\omega}{c_{o}}+0.5 .
$$

Thus, the solution at medium to long ranges can be represented by the finite series 


$$
\mathbf{p}(r, z, t)=-j \frac{2}{H} \sum_{n=1}^{M} \sqrt{\frac{2 \pi}{K_{n} r}} \sin \left(k_{z n} z_{o}\right) \sin \left(k_{z n} z\right) e^{j\left(\omega t-K_{n} r+\pi / 4\right)}
$$

Equation (3.13) was used to model the signal, jammer and replica fields.

\subsection{Signal Model}

This section describes the signals that are measured on the receiver hydrophones. The received waveforms contain two components: the signal component which radiates from the source, and noise composed of low rank interference and white noise. Since the problem is posed as a joint detection and estimation problem, the solution is formulated as a hypothesis test on the data. We assume that strong directional interference is always present. Each measurement of the directional interference is "low rank" provided it can be modeled as some linear combination of $r$ basis vectors or modes. Low rank is applicable whenever the number of modes is less than the number of sensors [9]. Throughout this research we assume that $r$ is known and represents the number of jammers. In addition, we assume that the interference is composed of the low-rank component plus white Gaussian noise. Therefore, if the data consists of only interference, the $k^{\text {th }}$ measurement is modeled as

$$
x_{k}=n_{k}=\sum_{m=1}^{r} a_{m}^{k} e_{m}+w_{k}
$$

where $e_{m}$ are the $n \times 1$ vectors which are the basis from which the low rank component is generated, $a_{m}^{k}$ are scalars which model an unknown phase and gain shift and $w_{k}$ is white, Gaussian background noise. The ambient noise $w_{k}$ is statistically independent snapshot to 
snapshot and is distributed as multivariate complex Gaussian [7] with zero mean and covariance matrix $\sigma^{2} I$. n represents the number of sensors in the sensor array. The vertical configuration of hydrophones are evenly spaced at 10 meters apart to avoid spatial aliasing.

If the source signal is present, and the $H_{1}$ hypothesis is true, then the $k^{\text {th }}$ measurement can be written as:

$$
x_{k}=\beta_{k} s\left(r_{o}, z_{o}\right)+\sum_{m=1}^{r} a_{m}^{k} e_{m}+w_{k}=\beta_{k} s\left(r_{o}, z_{o}\right)+E a_{k}+w_{k}
$$

$E a_{k}$ is the low rank interference, $\left\{\begin{array}{llll}e_{1} \mid & e_{2} \mid & \cdots & e_{r}\end{array}\right\}$, and is modeled as unknown deterministic with known rank $\mathrm{r}$. It may or may not be Gaussian in nature, therefore, $E$ is modeled as unknown deterministic. The columns of $E$ are the $r$ modal vectors that make up the low rank interference. As stated previously, $r$ corresponds to the number of jammer(s) present and is known. Physically, low rank interference is produced by surface noise or mechanically generated correlated noise fields. $\beta_{k}$ models an unknown gain and phase shift. $s\left(r_{o}, z_{o}\right)$ is the array output generated by the source, where depth and range are the unknown parameters. The strength of the source and noise components are unknown. Also, some a priori knowledge of the general search area for the source is necessary. Measurements form the data matrix $X=\left\{\begin{array}{llll}x_{1} & x_{2} & \cdots & x_{K}\end{array}\right\}$. K indicates the number of snapshots. 


\subsection{Summary}

Chapter Three presented an overview of wave theory as well as the motivation in choosing the Normal Mode Method to model the respective fields. The second purpose of Chapter Three was to present the signal models that were used in the research. A description of the model was included to give the reader a physical idea of what composes the model. This leads to the next chapter of the thesis, which presents the mathematical derivation of the GLRT processor. 


\section{CHAPTER FOUR}

\section{THE GENERALIZED LIKELIHOOD RATIO PROCESSOR}

\subsection{Introduction}

Chapter Four presents the derivation of the GLRT processor. This includes the mathematical and theoretical discussion of each step in the development of the GLRT processor. The first section of this chapter presents a brief background discussion of low rank processors.

\subsection{Background}

The performance limit of an array processor is generally set by its ability to handle strong interference. Spatially localized noise can be significant when compared to other system noises. Through the use of a low rank model to represent the directional interference we can account for this problem. Low rank modeling means that a spatial waveform can be modeled as a linear combination of $r$ linearly independent vectors where $r$ is less then the number of sensors. Sources of the low rank interference in an ocean environment include rain, wind noise, surface ships, and biologics. The interference could include intelligent countermeasures.

One method that has been proposed for adaptively detecting a signal in low rank interference is the Principle Component Inverse (PCI) method $[13,14,15,16,17]$. The form of the interference must be

$$
n=\sum_{k=1}^{r} a_{k} e_{k}+z
$$


where the $n \times 1$ vectors $\left\{e_{k}\right\}$ are the basis or "modes" which generate the low rank

interference. $\left\{a_{k}\right\}$ are scalars which correspond to the $k^{\text {th }}$ interference mode and $\mathrm{z}$ represents the background noise. The PCI method regards the interference as the "signal" to be enhanced. The next step is to apply reduced rank signal enhancement to estimate the interference, remove it from the data, and match filter the residual. The motivation behind the PCI method is the optimum receiver structure for detecting a signal in the presence of strong low rank interference and weak white Gaussian noise. This method can be compared to a null steerer where the low rank component is nulled out. The advantage of the PCI method over conventional methods is that it has a faster rate of adaptation. The limitation of the PCI method lies in the restrictive scenario that must occur. The signal that is contained in the data set must be weaker then the low rank interference or absent; otherwise, it too is removed from the data.

Kelly proposed a Generalized Likelihood-Ratio Test (GLRT) for adaptively detecting a signal of unknown amplitude in zero-mean complex Gaussian noise with unknown covariance matrix. No structure is imposed on the interference covariance matrix. The test requires "primary" and "secondary" data sets [22]. The "secondary" data consists of interference-only data snapshots. Detection is done on the primary data which is a single snapshot of data. The GLRT is constructed by the ML estimates of the primary and secondary data. Our research is an extension of Kirsteins' development of the mathematical models and algorithms for real random variables applied to low-rank signal processing.

\subsection{Derivation of the GLRT}

The derivation of the GLRT is broken down into two sections. The first section assumes that the mean of the hypothesis, source, source power, and covariance matrix are 
known. This assumption is necessary to form the basic likelihood ratio (LR) detector. However, in practical situations, these quantities are not known, and a generalized likelihood-ratio test (GLRT) can be applied to detect and localize the source. Thus, the second section of the derivation assumes that the mean of the hypothesis, source, source power, and covariance matrix are unknown quantities.

\subsubsection{Optimum Detector: Known source location, source power, and noise covariance matrix}

The GLRT is derived for the data matrix $X$. We want to decide between hypotheses $H_{o}$ and $H_{1}$. We assume that the probability of the two hypotheses are equal; therefore, the maximum likelihood receiver is the likelihood ratio test [9]. The LR is a ratio of the probabilities of $X$ in the cases of $H_{o}$ and $H_{1}$. Therefore, we begin the derivation by determining the probability density functions for the two hypotheses.

In the $H_{1}$ model, the data consists of the signal, low rank component, and white Gaussian noise (WGN):

$$
x_{k}=\beta_{k} s\left(r_{o}, z_{o}\right)+\sum_{m=1}^{r} a_{m}^{k} e_{m}+w_{k}
$$

where $x_{k}$ is the data vector and $\beta_{k}$ models an unknown gain and phase shift. The scalar weighting coefficient is represented by $a_{m}^{k}$ and $e_{m}$ are the array pressure fields produced by the discrete noise sources; they are modeled as unknown, deterministic quantities. The white Gaussian noise (WGN) is represented by $w_{k}$. The dimensions for the vectors found in equation (4.2) are 


$$
\begin{array}{lll}
x_{k}: & n \times 1, & k=1,2, \ldots, K \\
w_{k}: & n \times 1, & k=1,2, \ldots, K \\
a_{m}: & 1 \times 1, & m=1,2, \ldots, r \\
e_{m}: & n \times 1, & m=1,2, \ldots, r \\
\beta_{k}: & 1 \times 1, & k=1,2, \ldots, K \\
s: & n \times 1 &
\end{array}
$$

where $r$ is the number of directional jammers, $n$ is the number of sensors in the hydrophone array and $\mathrm{K}$ is the number of snapshots. The next step is to partition the vectors. Once this is complete equation (4.2) can be rewritten in the following form:

$$
x_{k}=\beta_{k} s\left(r_{o}, z_{o}\right)+\sum_{m=1}^{r} a_{m}^{(k)} e_{m}+w_{k}=\beta_{k} s\left(r_{o}, z_{o}\right)+E a^{(k)}+w_{k}
$$

The noise covariance matrix is $\sigma^{2} I=C_{n}$, where $\sigma^{2}$ is unknown and the mean is $E a_{k}+\beta_{k} s$. The multivariate Gaussian PDF of the $n \times 1$ complex random vector $y$ is

$$
f(y)=\frac{1}{\pi^{n}\left|C_{n}\right|} \exp \left\{-(y-\bar{y})^{H} C_{n}^{-1}(y-\bar{y})\right\}
$$

Substituting the mean of $x_{k}$ and the determinant for $C_{n}$ into the definition given in equation (4.5) yields

$$
f\left(x_{k} \mid E a_{k}+\beta_{k} s\right)=\frac{1}{\pi^{n} \sigma^{2 n}} \exp \left\{-\frac{1}{\sigma^{2}}\left(x_{k}-E a_{k}-\beta_{k} s\right)^{H}\left(x_{k}-E a_{k}-\beta_{k} s\right)\right\}
$$

Equation (4.6) represents the pdf of an individual snapshot of data. We assume that the snapshots are independent and identically distributed (iid). Therefore, the probability density function for the data matrix $X$ under the $H_{1}$ hypothesis is 


$$
f\left(X \mid H_{1}\right)=\frac{1}{\pi^{n K} \sigma^{2 n K}} \exp \left\{\frac{-1}{\sigma^{2}} \sum_{k=1}^{K}\left(x_{k}-E a_{k}-\beta_{k} s\right)^{H}\left(x_{k}-E a_{k}-\beta_{k} s\right)\right\} .
$$

However, equation (4.7) does not represent the final form we desire for the density function. To find a more convenient expression, we begin by closely looking at the summation portion of equation (4.7). Recall from equation (4.4) that $x_{k}-E a_{k}-\beta_{k} s=w_{k}$. Placing the Gaussian white noise, $w_{k}$ into the summation and then expanding, the following results

$$
\sum_{k=1}^{K}\left(w_{k}\right)^{H}\left(w_{k}\right)=\left(w_{1}\right)^{H}\left(w_{1}\right)+\left(w_{2}\right)^{H}\left(w_{2}\right)+\ldots+\left(w_{K}\right)^{H}\left(w_{K}\right)
$$

where $w$ in the above equation is in vector form. Thus, for each $w$ term the following property would hold

$$
w_{1}^{H} w_{1}=\sum_{m=1}^{n} w_{1}(m) w_{1}^{*}(m)=\sum_{m=1}^{n}\left|w_{1}(m)\right|^{2}
$$

Now, placing this property for each case in the summation, equation (4.8) becomes

$$
\sum_{k=1}^{K}\left(w_{k}^{H}\right)\left(w_{k}\right)=\sum_{k=1}^{K} \sum_{m=1}^{n}\left|w_{k}(m)\right|^{2}=\sum_{m=1}^{n} \sum_{k=1}^{K}\left|w_{k}(m)\right|^{2}
$$

As mentioned earlier, the vectors must be partitioned to determine the probability density function (pdf) for the data matrix $X$ under the $H_{1}$ hypothesis. Thus, for clarification the dimensions of the matrices are 


$$
\begin{aligned}
& X_{n \times K}=\left[\begin{array}{llll}
x_{1} \mid & x_{2} \mid & \cdots & \mid x_{K}
\end{array}\right] \\
& E_{n \times r}=\left[\begin{array}{llll}
e_{1} \mid & e_{2} \mid & \cdots & \mid e_{r}
\end{array}\right] \\
& A_{r \times K}=\left[\begin{array}{llll}
a_{1} \mid & a_{2} \mid & \cdots & \mid a_{K}
\end{array}\right] \\
& W_{n \times K}=\left[\begin{array}{llll}
w_{1} \mid & w_{2} \mid & \cdots & \mid w_{K}
\end{array}\right] \\
& b_{K \times 1}=\left[\begin{array}{lll}
\beta_{1}, \beta_{2}, \cdots, \beta_{K}
\end{array}\right]^{T} .
\end{aligned}
$$

This leads to reformulating equation (4.4) into the following convenient vector/matrix expression:

$$
X=s b^{T}+E A+W
$$

Finally, using the partitioned matrices, the Frobenius norm definition, and equations (4.8), (4.11), and (4.13), we find that the pdf for the data assuming $H_{1}$ is

$$
p\left(X \mid H_{1}\right)=\frac{\exp \left(\frac{-1}{\sigma^{2}}\left\|X-E A-s b^{T}\right\|_{F}^{2}\right)}{\pi^{n K} \sigma^{2 n K}},
$$

where $\mathrm{F}$ denotes the Frobenius norm.

The derivation for $p\left(X \mid H_{o}\right)$ is accomplished in a similar manner. Since the signal is not present in the null hypothesis, the data is normally distributed with a mean of $E a_{k}$. By applying the same concepts as were applied in the $H_{1}$ case it can be found that the resulting pdf is

$$
p\left(X \mid H_{o}\right)=\frac{\exp \left(\frac{-1}{\sigma^{2}}\|X-E A\|_{F}^{2}\right)}{\pi^{n K} \sigma^{2 n K}} .
$$

Assuming E, A, b, s, and $\sigma^{2}$ are known, the optimum detector is the likelihood ratio 


$$
z=\frac{p\left(X \mid H_{1}\right)}{p\left(X \mid H_{o}\right)}=\frac{\frac{1}{\pi^{n K} \sigma^{2 n K}} \exp \left\{\frac{-1}{\sigma^{2}}\left\|X-E A-s b^{T}\right\|_{F}^{2}\right\}}{\frac{1}{\pi^{n K} \sigma^{2 n K}} \exp \left\{\frac{-1}{\sigma^{2}}\|X-E A\|_{F}^{2}\right\}}
$$

which is the foundation for the GLRT [4].

\subsubsection{Unknown source power and noise covariance matrix}

In a practical situation, $\mathrm{E}, \mathrm{A}, \mathrm{s}, \mathrm{b}$, and $\sigma^{2}$ are unknown. While we may have some general a priori information on the general source location, we usually have no knowledge of the source, jammer(s) or WGN power. Since E, A, b, and $\sigma^{2}$ are not known, we propose a generalized likelihood-ratio test (GLRT) to detect and localize the signal. The GLRT is constructed by replacing the unknown signal amplitude and covariance matrix with their maximum likelihood (ML) estimates [9]:

$$
z=\frac{\max _{E, A, b, \sigma^{2}} \frac{1}{\sigma^{2 n K}} \exp \left\{\frac{-1}{\sigma^{2}}\left\|X-E A-s b^{T}\right\|_{F}^{2}\right\}}{\max _{E, A, \sigma^{2}} \frac{1}{\sigma^{2 n X}} \exp \left\{\frac{-1}{\sigma^{2}}\|X-E A\|_{F}^{2}\right\}} .
$$

In the next two sections, we will show how to obtain a closed-form solution for $z$.

\subsubsection{Maximizing the GLRT denominator}

The $z$ statistic is obtained by solving two separate problems. The denominator in equation (4.16) is the maximum value of the likelihood function after the unknowns are replaced by their ML estimates. Therefore, we need to maximize the function found in the 
denominator with respect to the unknowns $\mathrm{E}, \mathrm{A}$, and $\sigma^{2}$. The maximization of the function is accomplished by minimizing the exponential term and determining the ML estimate of $\sigma^{2}$. Thus, we begin by minimizing $\|X-E A\|_{F}^{2}$, where EA is an unknown rank $r$ matrix. Now, as previously discussed EA is a low rank approximation to the data matrix $X$. To minimize $\|X-E A\|_{F}^{2}$ we must approximate the data matrix $X$ by the estimate of the lower rank matrix EA. This is accomplished by applying theorems derived by Gabriel [8]. We begin by describing the singular value decomposition of $X$ :

$$
X=\sum_{\alpha=1}^{n} \lambda_{\alpha} p_{\alpha} q_{\alpha}^{\prime}
$$

where $\lambda_{1} \geq \lambda_{2} \geq \ldots \lambda_{n}$ are the singular values of $X$ and the $p_{\alpha}{ }^{\prime}$ s are the left singular vectors and the $q_{\alpha}^{\prime \prime}$ s are the right singular vectors. The rank $r$ matrix which minimizes $\|X-E A\|_{F}^{2}$ is

$$
\hat{E A}=\sum_{\alpha=1}^{r} \lambda_{\alpha} p_{\alpha} q_{\alpha}^{\prime}
$$

and is also the ML estimate of EA. This estimate will effectively give the best low rank approximation to $X$. In order to compute $\min \|X-E A\|_{F}^{2}$, we compute

$$
X-\hat{E A}=\sum_{\alpha=1}^{n} \lambda_{\alpha} p_{\alpha} q_{\alpha}^{\prime}-\sum_{\alpha=1}^{r} \lambda_{\alpha} p_{\alpha} q_{\alpha}^{\prime}=\sum_{\alpha=r+1}^{n} \lambda_{\alpha} p_{\alpha} q_{\alpha}^{\prime},
$$

where $\lambda_{r+1}, \ldots, \lambda_{n}$ are the singular values of $X-\hat{E A}$, and $p_{r+1}, \ldots, p_{n}$ and $q_{r+1}^{\prime}, \ldots, q_{n}^{\prime}$ are the singular vectors of $X-\hat{E A}$. Now, in Gabriel's paper the results for bilinear least squares approximation is stated in four alternative forms. By applying his results to our problem we find that the final form can be stated as 


$$
\min _{E A}\|X-\hat{E A}\|_{F}^{2}=\sum_{k=r+1}^{n} \lambda_{k}^{2}
$$

The residual seen in equation (4.20) is the result of effectively estimating the directional interference and removing it from the data collected. This will suppress the noise and physically will be seen in the lower sidelobes in the plots, which in turn allows a better estimate of the true location of the source. To complete this portion of the derivation the $\mathrm{ML}$ estimate of the variance must be determined. This is accomplished by first taking the natural log of the pdf

$$
\ln f\left(X \mid H_{o}\right)=-n K \ln (\pi)-n K \ln \left(\sigma^{2}\right)-\frac{1}{\sigma^{2}}\|X-E A\|_{F}^{2}
$$

Then, compress the likelihood function by substituting the MLE of $E A$ into equation (4.21). Next, take the derivative with respect to $\sigma^{2}$ and set it equal to zero

$$
\frac{-n K}{\sigma^{2}}+\frac{1}{\sigma^{4}}\|X-\hat{E A}\|_{F}^{2}=0 .
$$

Therefore, the $\mathrm{ML}$ estimate of the noise variance is

$$
\hat{\sigma}^{2}=\frac{1}{n K}\|X-\hat{E A}\|_{F}^{2},
$$

substituting equation (4.20) into equation (4.23) yields

$$
\hat{\sigma}^{2}=\frac{1}{n K} \sum_{k=r+1}^{n} \lambda_{k}^{2} .
$$


Thus, placing the ML estimates found in equations (4.20) and (4.24) into the denominator of equation (4.16), the result is

$$
\max _{E, A, \sigma^{2}} p\left(X \mid H_{o}\right)=\frac{1}{\left(\frac{1}{n K} \sum_{k=r+1}^{n} \lambda_{k}^{2}\right)^{n K}} \exp \{-n K\} .
$$

\subsubsection{Maximizing the GLRT numerator}

The analysis for the numerator is similar except for the linear term $s b^{H}$. The ML estimate for $E, A, b$, and $\sigma^{2}$ must be derived. To find the $\mathrm{ML}$ estimate for the mean $\left(E A-s b^{T}\right)$ a least squares theorem for joint linear and bilinear fit is used. We approach the numerator problem as previously discussed, where to maximize the function we minimize the exponential term. Thus, applying Gabriel's results

$$
\min _{E(n \times r)} \min _{A(n \times K)} \min _{b(1 \times K)}\left\|X-s b^{T}-E A\right\|_{F}^{2}=\min _{E A(n \times K)}\|\tilde{X}-\hat{E A}\|_{F}^{2}
$$

where

$$
\tilde{X}=(I-P) X
$$

and $I-P$ is the orthogonal projection matrix $P^{\perp}$ where

$$
P=\frac{s(r, z) s(r, z)^{H}}{\|s(r, z)\|^{2}}
$$


The next step is to find the best low rank approximation of $\tilde{X}$. This is achieved through similar methods as seen in equations (4.19) and (4.20)

$$
\tilde{X}-\hat{E A}=\sum_{\gamma=1}^{n} \tau_{\gamma} p_{\gamma} q_{\gamma}^{\prime}-\sum_{\gamma=1}^{r} \tau_{\gamma} p_{\gamma} q_{\gamma}^{\prime}=\sum_{\gamma=1+r}^{n} \tau_{\gamma} p_{\gamma} q_{\gamma}^{\prime}
$$

Thus, applying Gabriel's results we find

$$
\min _{E, A, b}\left\|\tilde{X}-\hat{E A}-s \hat{b}^{T}\right\|_{F}^{2}=\sum_{k=r+1}^{n} \tau_{k}^{2}
$$

where $\tau_{k}$ are the singular values of $P^{\perp} X$. Finally, applying the similar procedure used in the denominator case the ML estimate of the variance is

$$
\hat{\sigma}^{2}=\frac{1}{n K} \sum_{k=r+1}^{n} \tau_{k}^{2}
$$

It is important to note that when doing a joint linear and bilinear fit, the order in which the fitting is performed is important. If we would have first fitted the bilinear terms and then the linear terms the result would not be a joint least squares fit.

Finally, substituting equations (4.30) and (4.31) into the numerator of equation (4.16) yields

$$
\max _{E, A, \sigma^{2}, b} p\left(X \mid H_{1}\right)=\frac{1}{\left(\frac{1}{n K} \sum_{k=r+1}^{n} \tau_{k}^{2}\right)^{n K}} \exp \{-n K\}
$$

Finally, substituting the results seen in equations (4.25) and (4.32) into equation (4.16) yields, 


$$
z=\frac{\max _{E, A, b, \sigma^{2}} \frac{1}{\sigma^{n K}} \exp \left\{\frac{-1}{\sigma^{2}}\left\|X-E A-s b^{T}\right\|_{F}^{2}\right\}}{\max _{E, A, \sigma^{2}} \frac{1}{\sigma^{n K}} \exp \left\{\frac{-1}{\sigma^{2}}\|X-E A\|_{F}^{2}\right\}}=\frac{\sum_{k=r+1}^{n} \lambda_{k}^{2}}{\sum_{k=r+1}^{n} \tau_{k}^{2}} .
$$

The GLRT simplifies to the ratio of the modeling errors of the respective hypotheses. By estimating and removing the low rank interference we are left with a direct estimate of the white noise in both hypothesized data models.

\subsection{GLRT Implementation for Localization}

The preceding derivation assumed the source signal $\underline{\mathbf{s}}$ is known, however, in practical situations the source signal is unknown. The only known is a basic idea of where to search for the source.

Therefore, now that the mathematical basis for the GLRT has been discussed, comprehension of the practical implementation is necessary. Through the use of the propagation models we can generate a signal field at each element of our receiver array for any given source location. The complex acoustic field is modeled as a function of source location and is called the "replica field". The replica field is computed for a source placed at hypothesized range and depth locations. This information is then incorporated into the projection matrix $P$. The $z$ statistic that is computed through the use of the GLRT is a function of range and depth. An "ambiguity surface" is produced by calculating the test statistic over a trial set of hypothesized source ranges and depths. Peaks indicate the estimates of the location of strong directional signal sources. If the peak is greater than a predetermined threshold, we assert that a point acoustic source is present at that location. 
In summary, we propose the following new algorithm for matched-field processing:

\section{Off-line steps:}

1. Establish appropriate signal model for "low-rank": interference plus background.

2. Specify problem geometry, (i.e. number of sources, search domain, array geometry, and etc,)

3. Generate replica field data for projection matrix.

4. Select detector threshold.

\section{On-line steps:}

5. Update projection matrix from replica field data.

6. Calculate $z(\theta)$ according to equation (4.33) [4].

7. If $z(\theta)$ is greater than the predetermined threshold, we assert source is detected at location $\theta$.

\subsection{Summary}

This chapter included a detailed analysis of the GLRT derivation. The key to the GLRT is that a noise model has been directly introduced into the signal processing. By modeling the noise we can effectively estimate it and remove it from the data matrix. The estimation and removal of the noise suppresses the sidelobes and provides distinct peaks that indicate the source and jammer(s) location. In addition, signal-free training data is not required as in many adaptive signal processing algorithms. The decision process incorporated into the GLRT introduces detection capabilities to MFP. The Bartlett and MLM estimators are not specifically designed for detection. Finally, the step by step procedure for implementing the GLRT was discussed in the final section of this chapter. 
The results of the GLRT processor and comparisons to previous methods are included in Chapter Five. 


\section{CHAPTER FIVE}

\section{RESULTS}

\subsection{Introduction}

Chapter Five describes representative results from the GLRT. The first section describes the testing conditions of the ocean environment in which the research was conducted. Chapter Five presents three cases that directly compare the GLRT to the Bartlett and MLM processors. Plots of how the processors performed are included. The analysis of the GLRT is also discussed in this chapter. This section discusses why the output of the GLRT produces peaks at the corresponding estimates of the source and jammer(s) locations. Finally, the strengths and limitations of the GLRT are examined.

\section{$5.2 \quad$ Scenario}

Chapter Four discussed the complete GLRT derivation. The derivation included a detailed discussion on the two hypotheses that composed the GLRT. In this chapter we assume that one peak of the output corresponds to the estimated source location. We did not include plots which would represent the presence of no source; we assume the null hypothesis is eliminated. The GLRT was tested in a simulated shallow water environment. We chose an isospeed shallow water channel with a rigid bottom and a uniform sound velocity profile (SVP) [2]. Figure 5.1 is an illustration of the scenario that is studied in this chapter. The equation that is used to generate the signal field, the jammer field, and the replica vectors is

$$
p(r, z, t)=-\frac{2}{H} \sum_{n=1}^{M} \sqrt{\frac{2 \pi}{K_{n} r}} \sin \left(k_{z n} z_{o}\right) \sin \left(k_{z n} z\right) e^{-j K_{n} r} .
$$




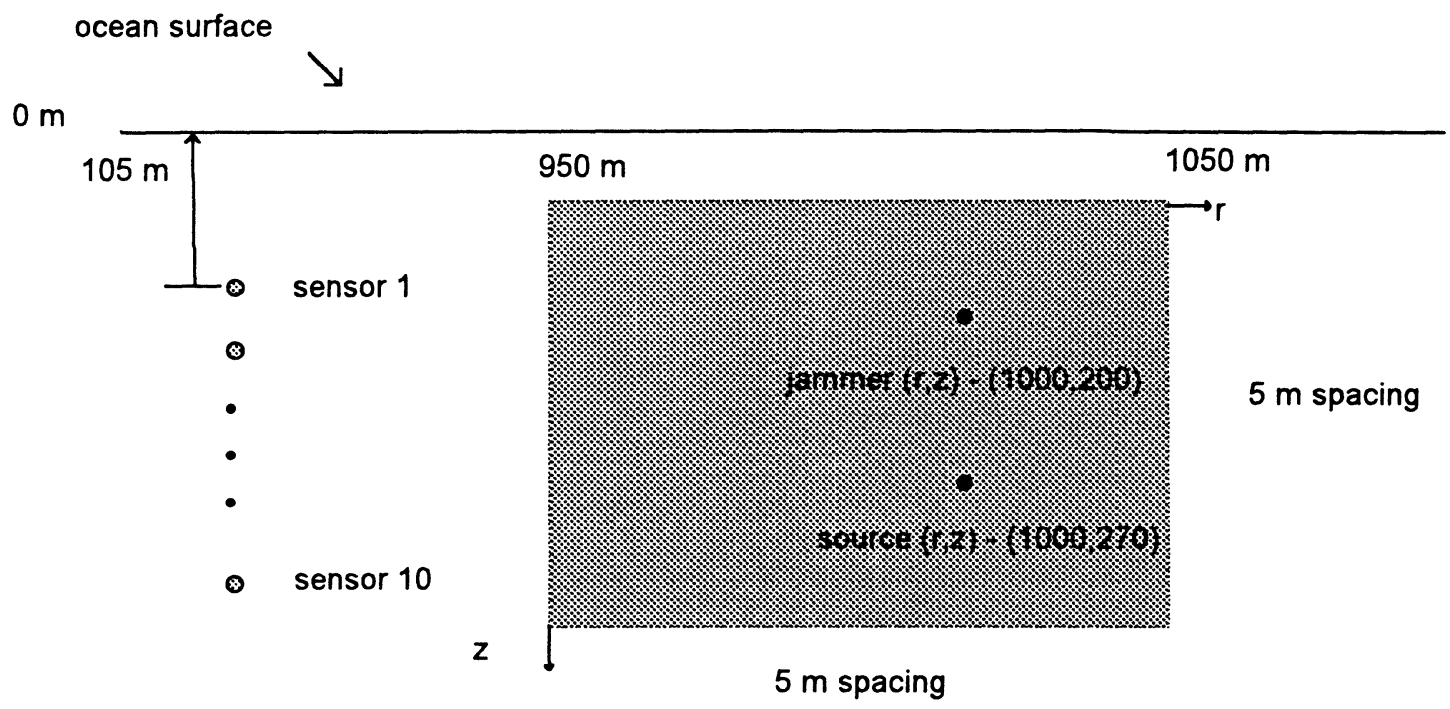

$500-m$

infinite subbottom

Figure 5.1 Geoacoustics of the environment used for simulations. 
$\mathrm{H}$ is the water depth and for all tests is set at 300 meters. $\left\{k_{z n}\right\}$ are the vertical wave numbers (eigenvalues) and are calculated by

$$
k_{z n}=(n-0.5) \pi / H
$$

for $n=1,2,3, \ldots, M .\left\{K_{n}\right\}$ are the horizontal wave numbers and are calculated by

$$
K_{n}=\sqrt{\left(\frac{\omega}{c}\right)^{2}-\left(k_{z n}\right)^{2}},
$$

where $\mathrm{c}$ is the speed of sound, 1500 meters $/ \mathrm{sec}$, and $\omega$ is the frequency set at $75 \mathrm{~Hz} . \mathrm{M}$ is the number of normal modes; $r$ is the range of the source, and $z_{o}$ is the source depth.

For purposes of demonstration, the signal power is $5 \mathrm{~dB}$ and the white noise is -10 dB. The strength of the jammer varies in each case. The window in which the ranges for the replica field are computed is 950 to 1050 meters. The true location of the source is set at a depth of 270 meters and at a range of 1000 meters. A jammer is located at a depth of 200 meters and at a range of 1000 meters. The number of snapshots is 100 and the number of sensors is 10 .

The following section presents three cases that show what occurs as the jammer strength varies. In each case the results include how the Bartlett, MLM and GLRT processors handle the scenario that is applied. All other parameters in each case remain the same. 


\subsubsection{Case 1: Jammer Power $=10 \mathrm{~dB}$}

In case one, the strength of the jammer is stronger then the source. The source is $5 \mathrm{~dB}$. These results are interesting due to the strength of the interference. We see in Figure 5.2 that the strong jammer causes spatial spectral leakage in the Bartlett processor which is manifest in the high sidelobes. The Bartlett processor can not effectively handle the strong interference. Therefore, the high sidelobes lead to ambiguous results, as to an accurate estimate of the source location. Examination of the data shows that the Bartlett estimator inaccurately estimates the location of the source. This is directly due to the processors inability to handle the strong interference.

The MLM processor results are illustrated in Figure 5.3. The MLM effectively suppresses the sidelobes and two distinct peaks denote estimates of the source and jammer locations. Unlike the Bartlett processor these are accurate estimates. The speed of this processor is slower then the Bartlett processor and the amplitude of the peaks are not significantly greater then the floor of plot. Although the MLM processor is not designed for detection, it is capable of detection but its performance would be poor.

Figure 5.4 represents the output of the GLRT processor. The GLRT processor produces two distinct peaks on the ambiguity surface plots. The GLRT processor is specifically designed for detection unlike the Bartlett and MLM processors. In this case the peaks that are seen in the plot are an accurate estimate of the source and jammer locations. The Bartlett and MLM results will continue to decrease as the strength of the jammer is increased. If the white noise power were to increase the results of all estimators would be degraded. 


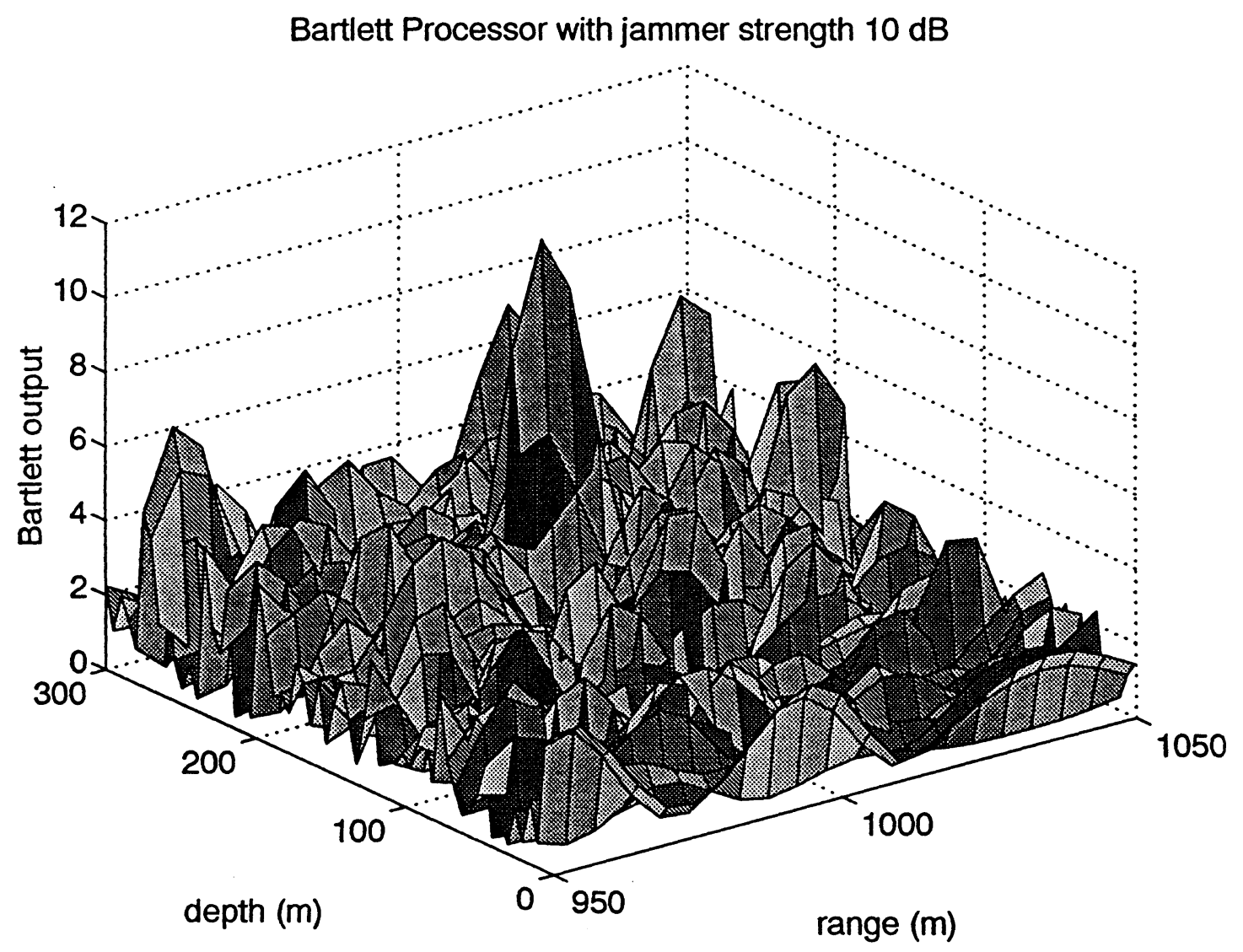

Figure 5.2 MFP Case 1: Bartlett Ambiguity Surface 


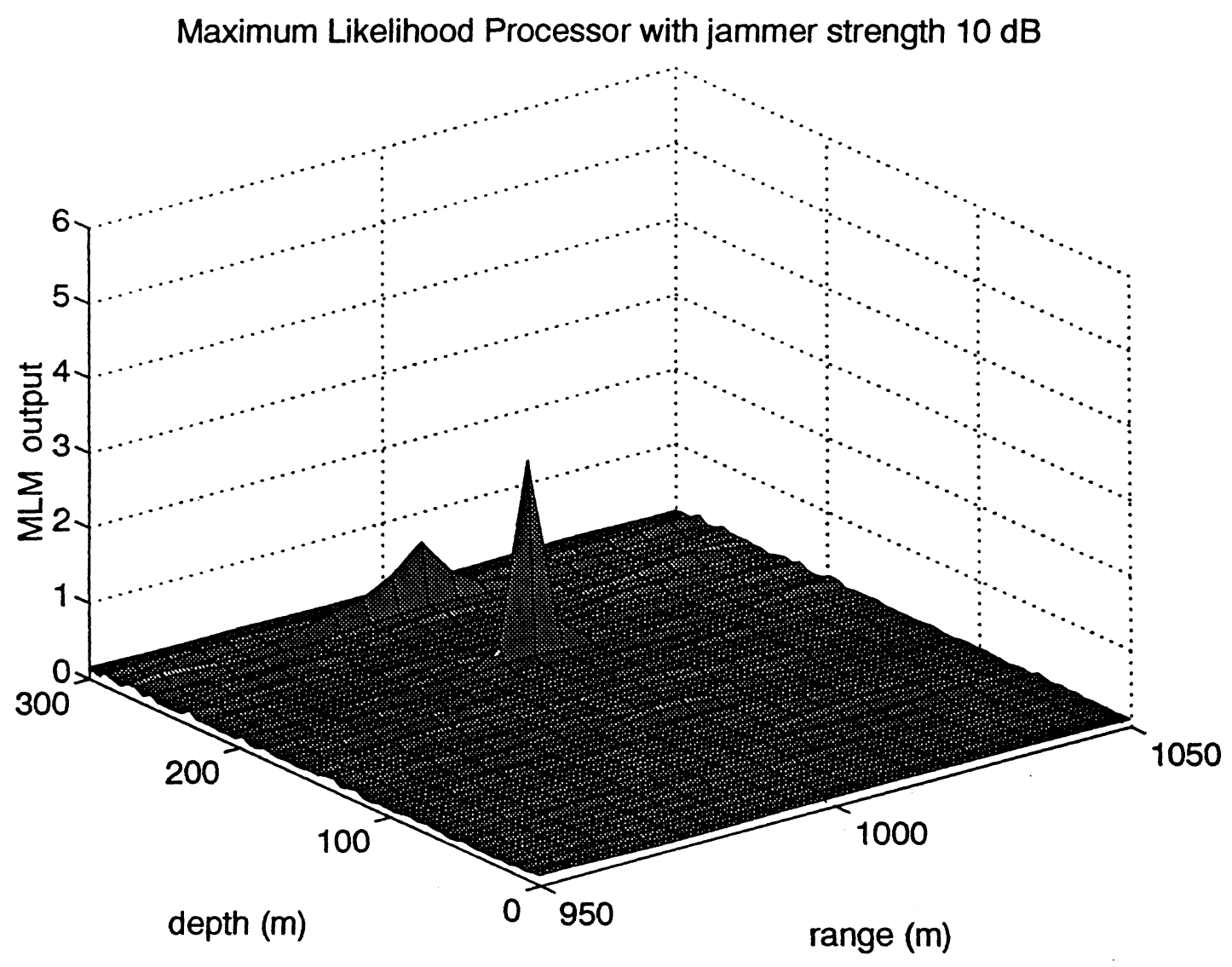

Figure 5.3 MFP Case 1: MLM Ambiguity Surface 


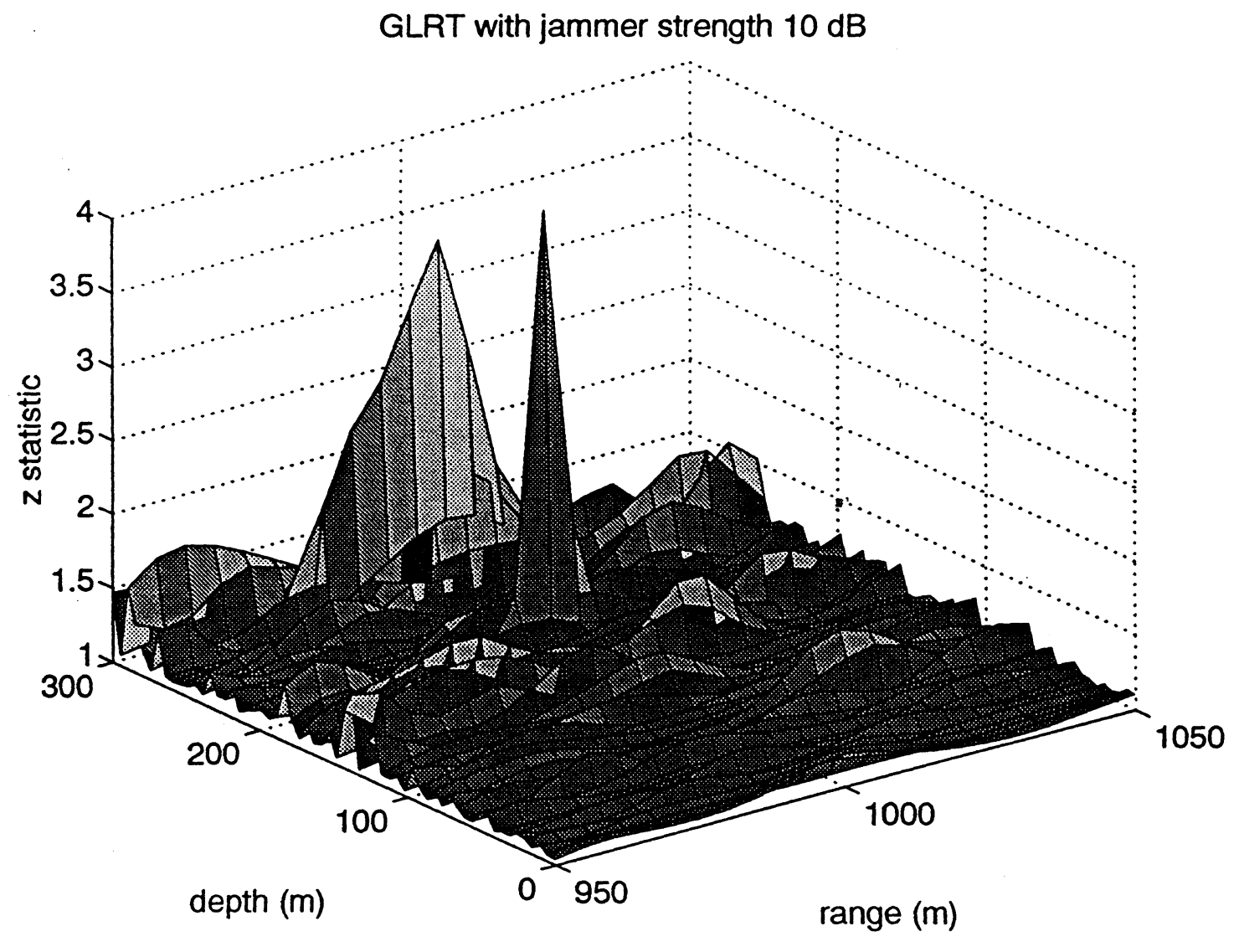

Figure 5.4 MFP Case 1: GLRT Ambiguity Surface 


\subsubsection{Case Two: Jammer Power $=0 \mathrm{~dB}$}

The Bartlett processor produces better results in this case as compared to case one. This is due to the strength of the directional interference decreasing. The plots of the Bartlett processor contained in this thesis show the high sidelobes that are inherent to the processor. However, in a practical sense, while the high sidelobes present problems, the data can be manipulated such that information could be extracted. Figure 5.6 shows that the MLM processor has produced two peaks. Comparing Figure 5.6 to Figure 5.7, which represents the output of the GLRT processor; it can be seen that the GLRT produces more distinct peaks when compared to the level of the floor of the plot. In this case the jammer to white noise power ratio is smaller. Therefore, as expected the degradation in the processors will occur. Recall the output of the GLRT is effectively a ratio of two white noise power estimates. Therefore, as the jammer to white noise power ratio decreases the results the GLRT processor will also produce less distinct peaks. In this case all three processors produce accurate results. 


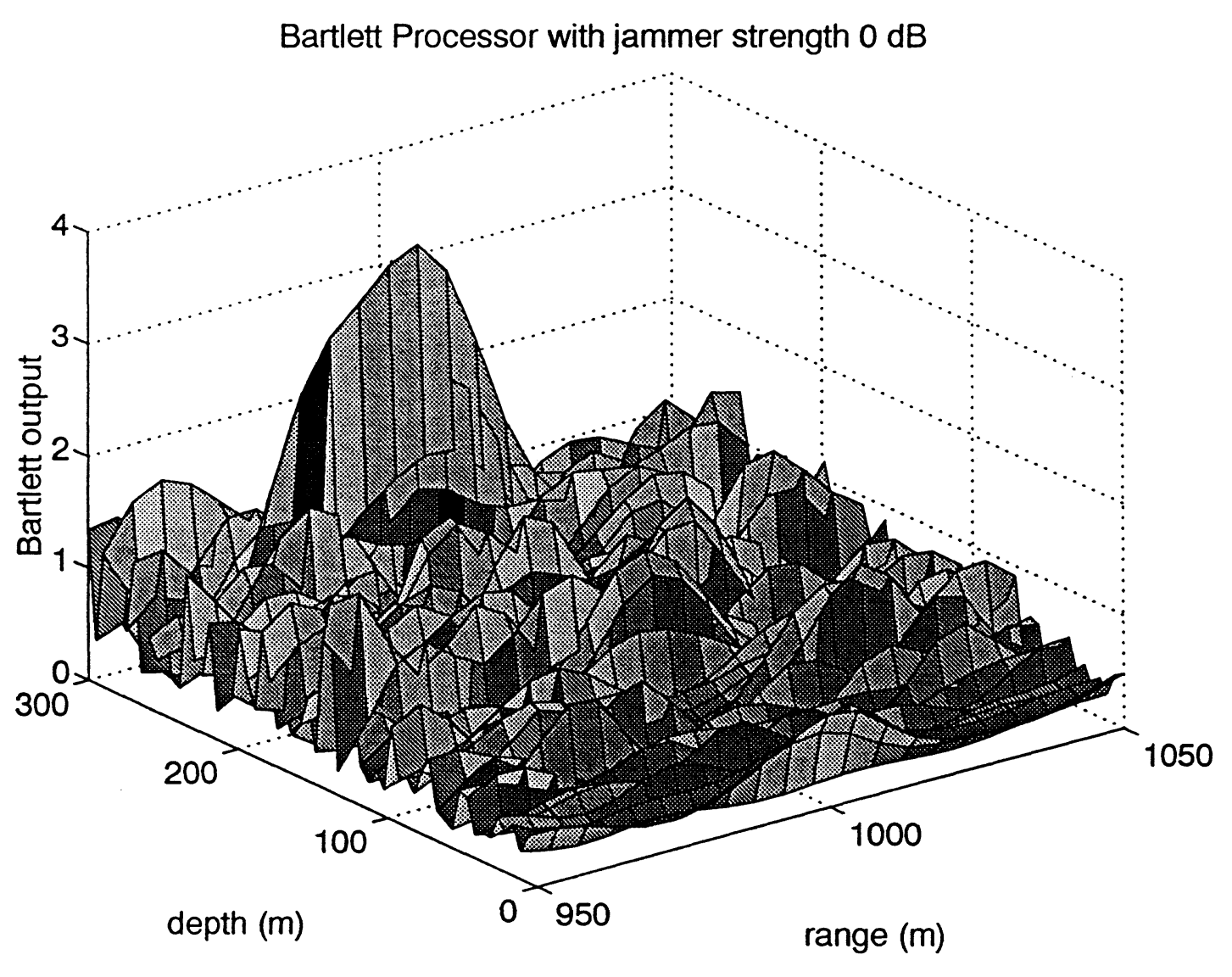

Figure 5.5 MFP Case 2: Bartlett Ambiguity Surface 


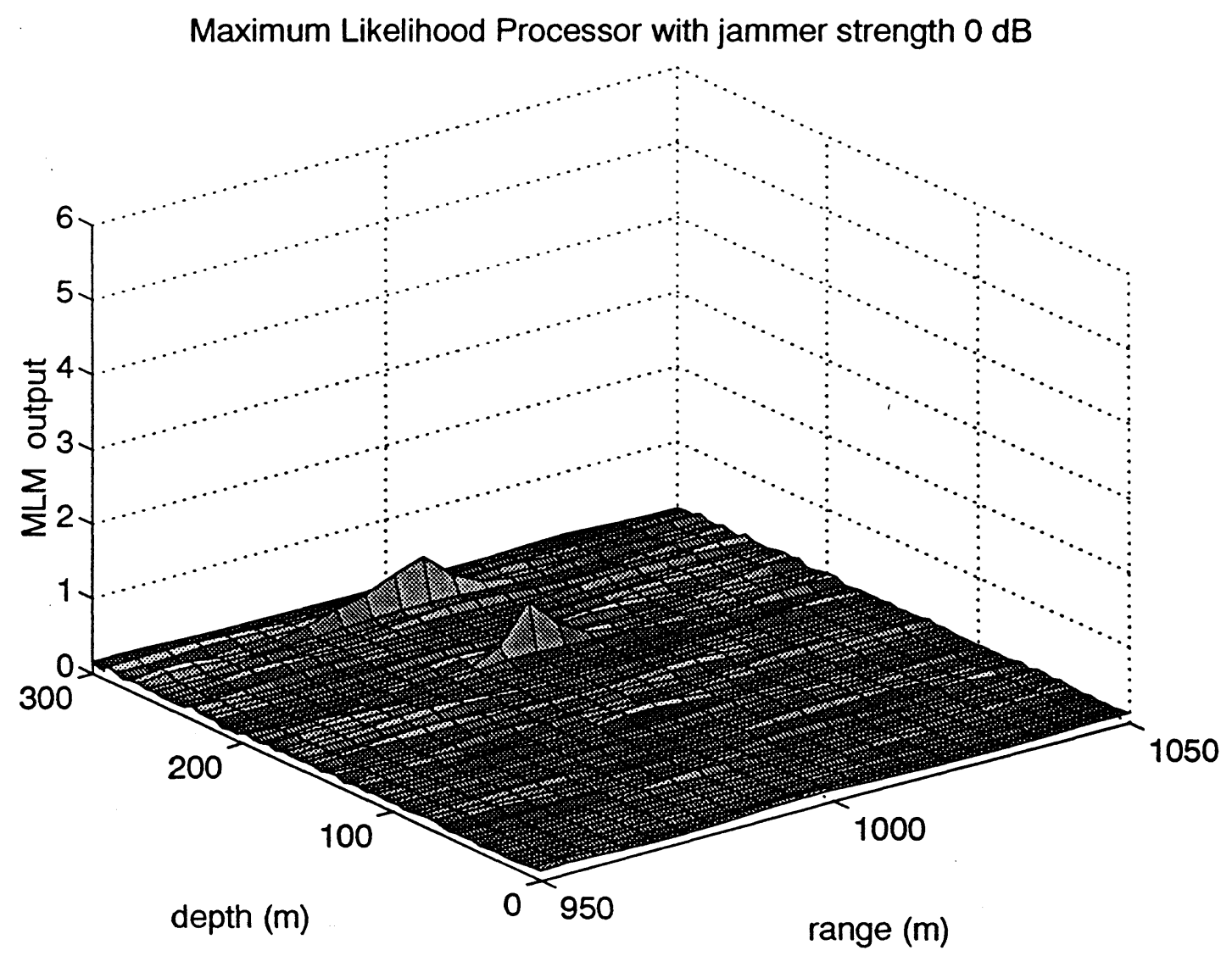

Figure 5.6 Case 2: MLM Ambiguity Surface 


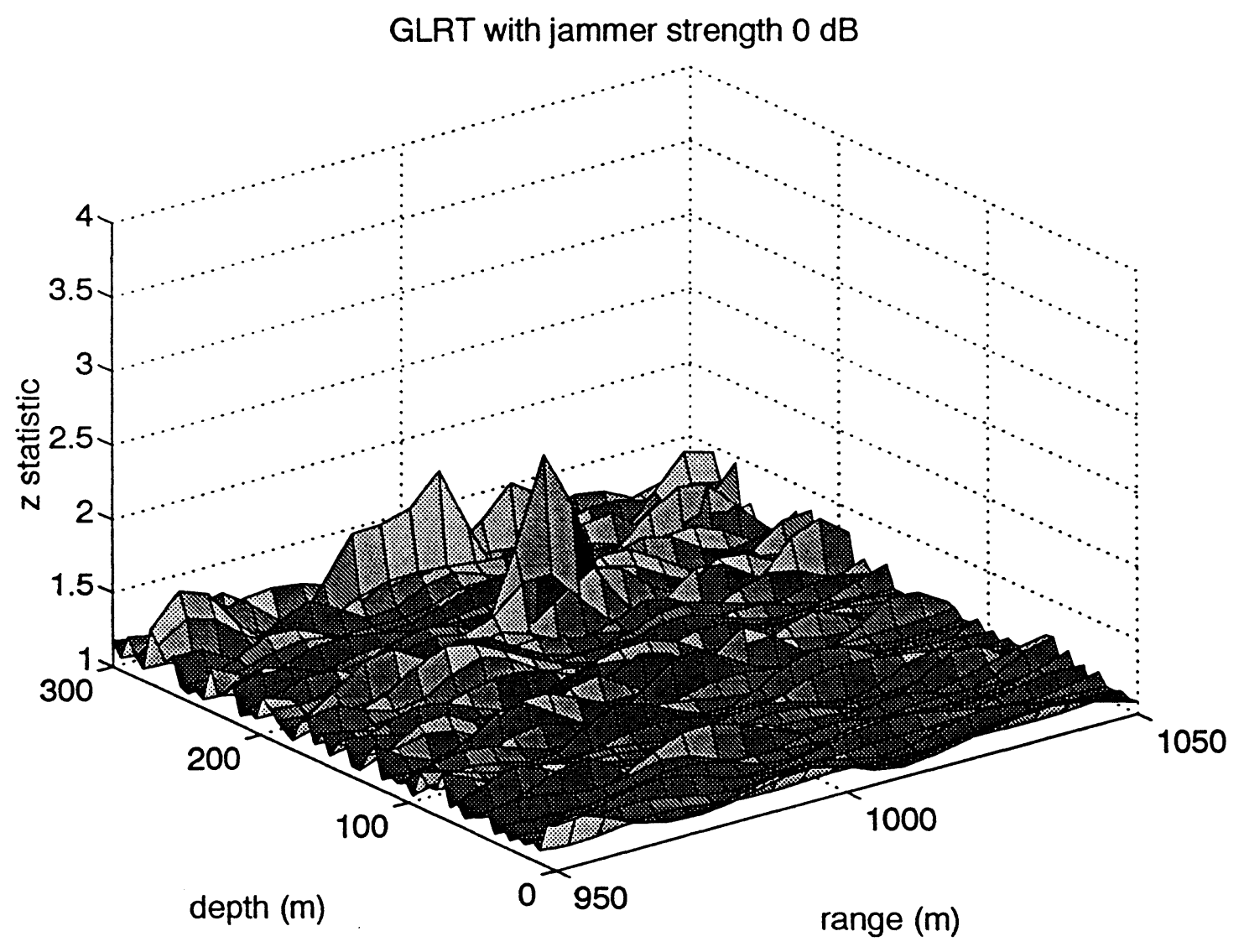

Figure 5.7 Case 2: GLRT Ambiguity Surface 


\subsubsection{Case Three: Jammer Power $=-\mathbf{1 0} \mathrm{dB}$}

This case presents the results of what occurs when the jammer strength is equivalent to the white noise. Therefore, in all three plots there is no distinct peak for the jammer. The Bartlett and MLM produce better results when the directional interference is not strong. This is seen in Figure 5.8 and Figure 5.9. Examining the GLRT processor we see that in this case the performance of the processor is poor. This case presents a limitation of the GLRT processor. When the directional interference is weak or comparable to the white noise power it is difficult to estimate the source location. This is understandable given the fact that the GLRT has been designed to handle strong interference. The GLRT inaccurately estimates the depth coordinate of the source location at 270 meters. The Bartlett and MLM processors produce accurate results. 


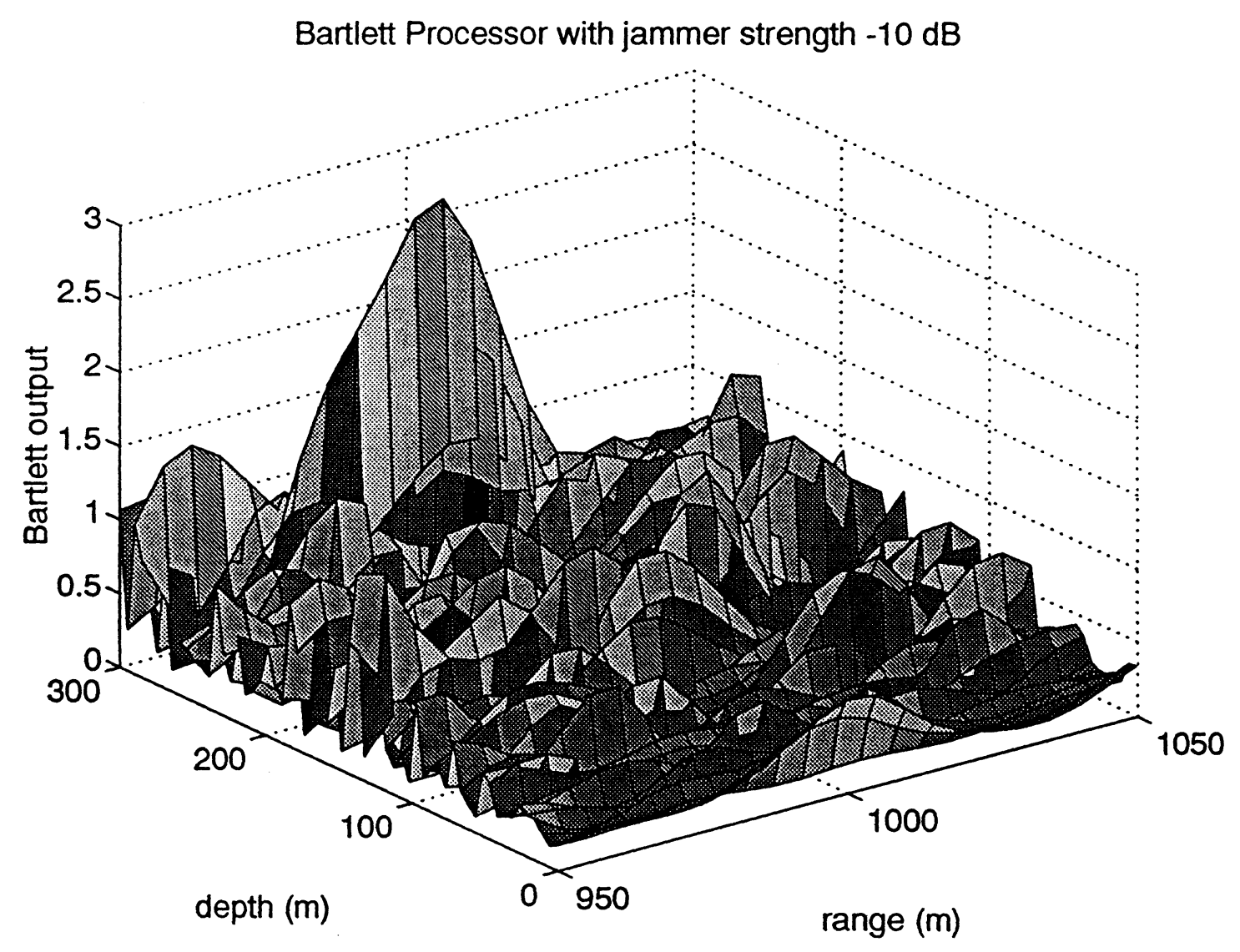

Figure 5.8 Case 3: Bartlett Ambiguity Surface 


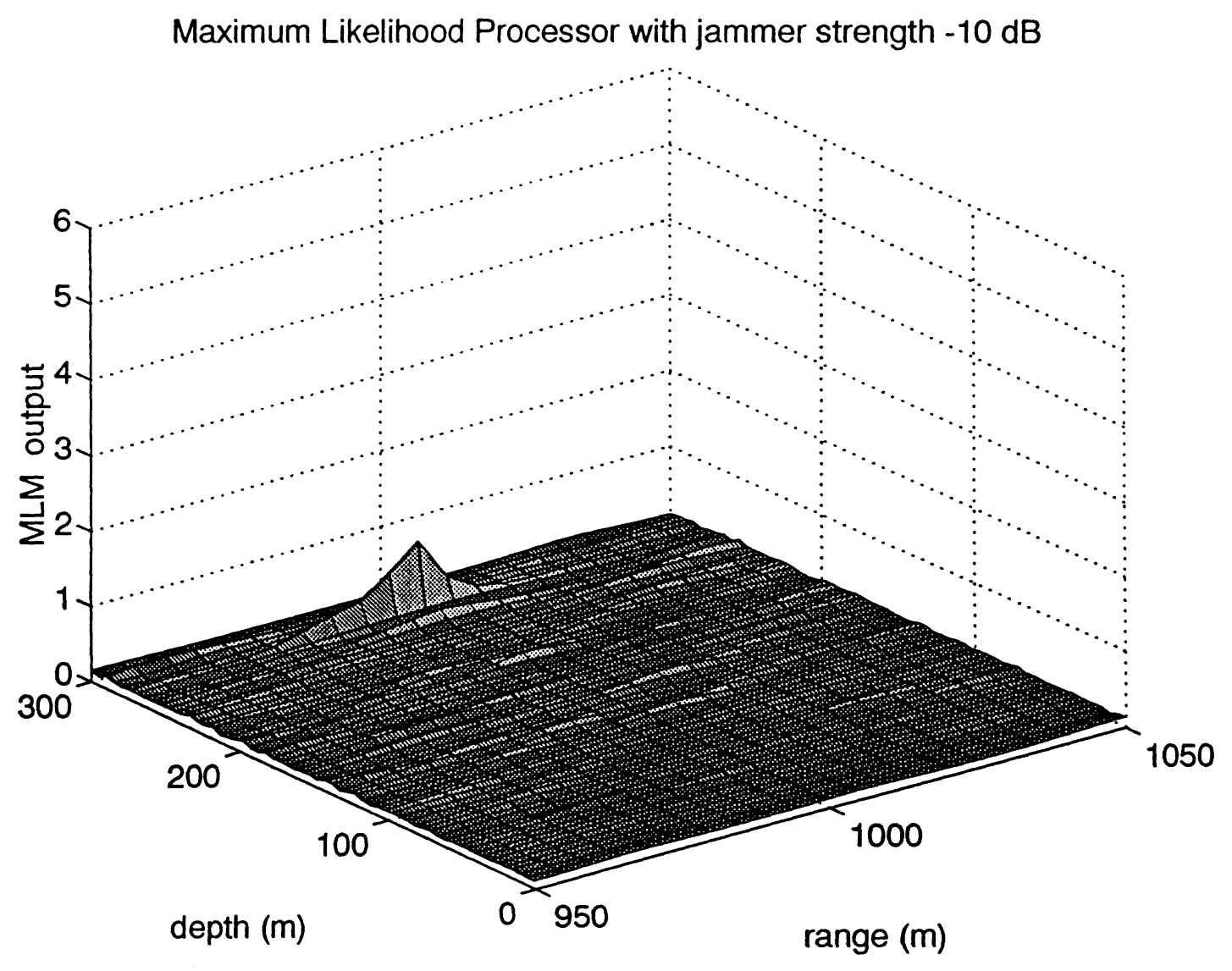

Figure 5.9 Case 3: MLM Ambiguity Surface 


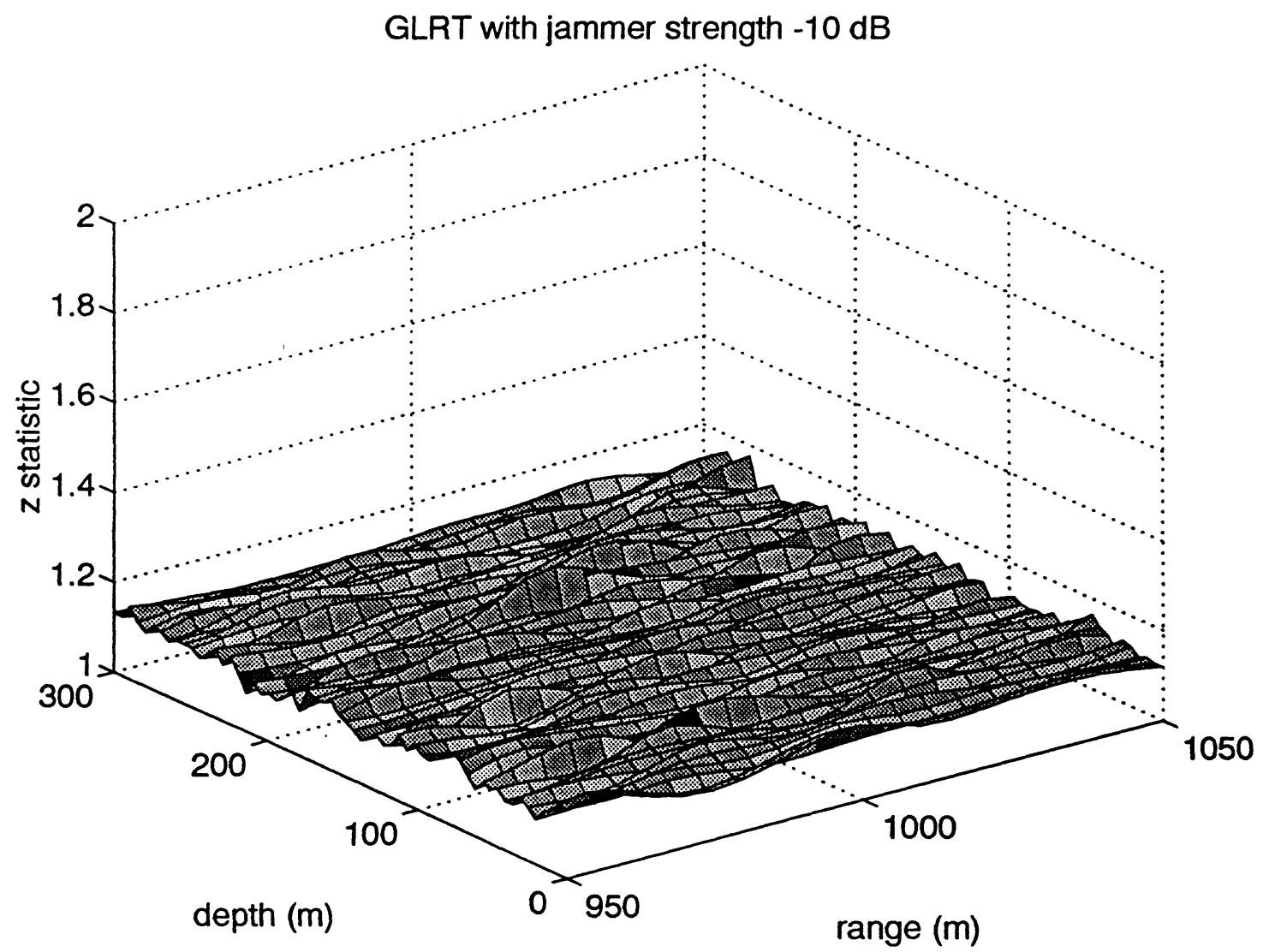

Figure 5.10 Case 3: GLRT Ambiguity Surface 


\subsection{GLRT Analyis}

Why do peaks occur in the output of the GLRT as the replica field sweeps across discrete sources? The answer is found in an eigenvalue analysis of the GLRT. Recall from the derivation of the GLRT that the output corresponds to a ratio of sums of the singular values squared of the respective hypotheses. Applying a well known math property, we can state that the singular values of $P^{\perp} X$ are equivalent to the eigenvalues of $P^{\perp}\left(\frac{1}{K} X X^{H}\right) P^{\perp}$. As the replica field sweeps through the environment it is effectively searching for a "match" or source. We need to consider how the eigenvalues of $P^{\perp}\left(\frac{1}{K^{\prime}} X X^{H}\right) P^{\perp}$ change as a function of $\theta$ as $\mathrm{K}$ becomes very large. We can gain first order information about the GLRT's performance by constructing an eigenstructure of $P^{\perp}\left(\frac{1}{K} X X^{H}\right) P^{\perp}$. We begin by making some assumptions about the data. First we assume that there is a large amount of data. Next, we assume that the noise structure is zero mean, independent and identically distributed (iid). Thus, we can say that the data matrix for the case of one jammer takes the familiar form

$$
X=s b^{H}+e a^{H}+W
$$

We can approximate that the cross terms of $\frac{1}{K} X X^{H}$ are zero and the result is denoted by $S_{o}$ as seen in equation (5.5).

$$
S_{o}=\sigma_{s}^{2} s s^{H}+\sigma_{j}^{2} e_{1} e_{1}^{H}+\sigma_{w}^{2} I
$$

In equation (5.5) $\sigma_{s}^{2}, \sigma_{j}^{2}$, and $\sigma_{w}^{2}$ represents the average power of the source, jammer, and white noise, respectively. 
Since we are searching for the source we eliminate the null hypothesis. There are two possible cases; a match of the source field and a mismatch. We begin by assuming the replica field is equal to $s$. Then

$$
P^{\perp} S_{o} P^{\perp}=-\sigma_{w}^{2} s s^{H}+\sigma_{j}^{2} e_{1} e_{1}^{H}+\sigma_{w}^{2} I
$$

where the assumption is that $s^{H} e_{1} \cong 0$. This implies that the jammer is not "close" to the signal. The largest eigenvalue of $P^{\perp} S_{o} P^{\perp}$ is $\sigma_{s}^{2}+\sigma_{w}^{2}$ (source is stronger then the jammer) and the smallest is zero. The second largest is $\sigma_{w}^{2}$ or an estimate of the white noise power. Recall from the derivation of the GLRT that the number of jammers denotes the number of eigenvalues that are not considered. In the case of one jammer we do not consider the largest eigenvalue, therefore, our analysis hinges on the result of the second largest eigenvalue. Now, when the source field is effectively matched the result is an accurate estimate of the white noise power. If the number of jammers is one, the largest eigenvalue is a close estimate of the jammer plus white noise power. However, this value is not considered by the GLRT processor. The remaining values are estimates of the white noise power except for the smallest value which is approximately zero (this is due to the matrix being rank $n-1)$. This small number in the denominator of the GLRT produces a large number in the output. This then indicates an estimate of a match or an estimate of the source location.

A mismatch occurs when the replica field is perpendicular to $s$ and $e_{1}$. If the source is stronger then the jammer the largest eigenvalue of $S_{o}$ is $\sigma_{s}^{2}+\sigma_{w}^{2}$ and the second largest eigenvalue is $\sigma_{j}^{2}+\sigma_{w}^{2}$. The smallest eigenvalue is $\sigma_{w}^{2}$. We see that the second largest eigenvalue in the case of $S_{o}$ is large. This implies that when the replica field is not matched to a source field the resulting eigenvalues are very large. This is due to a source 
field not being properly removed. This produces a high number in the denominator of the GLRT, thus, the output is small.

\subsection{Tradeoffs}

Throughout this thesis we have discussed not only the estimators currently being used in MFP (Bartlett and MLM) but also the motivation for a new processor (GLRT). Tradeoffs do exist between the estimators. For example, the Bartlett estimator is easy to implement and is a proven processor in practical situations. However, the output of the processor has high sidelobes. This leads to ambiguous results in the estimation of the source location. Therefore, the MLM processor was developed to handle the sidelobe problem inherent to the Bartlett processor. While the MLM estimator produces distinct peaks, which leads to a good estimate of the source location, it is a slower processor. Also, neither the Bartlett or MLM processors are specifically designed for detection.

The GLRT is specifically designed for detection. This is due to the design of the processor or the hypothesis test that is incorporated into the processor. Unlike the other estimators the GLRT processors can effectively handle strong interference, which is a key advantage. The capability to estimate and remove the noise is accomplished by incorporating a noise model into the processor.

Implementing the GLRT does have limitations. Currently, this processor is considerably slower then the other two processors. This could be improved by writing the code in a better language. Also, this processor needs to know exactly how many jammers are present. Future consideration will include giving the processor inaccurate estimates as to the number of jammers. Finally, this processor has not been proven in a practical situation. While preliminary results are encouraging, it has yet to be applied to a "real" ocean environment. 


\subsection{Summary}

This chapter presented various cases in which the performance of the GLRT was evaluated in direct comparison to processors currently used in MFP. These results proved that when strong interference is present the GLRT can effectively handle it and estimate the source location. The results also showed that even in cases when performance was degraded the GLRT processor was a better processor then the common estimators. However, unlike the other estimators, the GLRT is not a power estimator. The analysis on why peaks occur in the output of the GLRT was also presented in this chapter. Finally, the benefits and limitations of the GLRT processor were discussed in this chapter. 


\section{CHAPTER SIX}

\section{CONCLUSIONS}

\subsection{Conclusions}

This thesis presents a new technique for localizing and detecting sources in the presence of strong low rank interference. In Chapter Five a direct comparison of the GLRT processor to the current estimators, the Bartlett and Maximum Liklihood Method (MLM) was presented. The benefits and limitations of the GLRT processor were also discussed. However, considering the limitations mentioned, it is still a better estimator then what is currently available in the presence of strong interference.

At the beginning of the thesis our goals of achieving detection and localization in the presence of strong directional interference were presented. We successfully demonstrated the GLRT is a viable concept that can be applied to detecting and localizing a source in the presence of strong interference. Also, while the present methodologies can achieve distinct peaks, they are not specifically designed for detection.

\subsection{Future Work}

The next step in the research would be to do threshold tests on the GLRT. Future work should also include applying the GLRT to actual data. Changing the boundary conditions such as the SVP and the ocean environment to a more complex structure would prove interesting. Also, research could include studying more complex noise models. In our research we were aware of all the environmental parameters. Studying the effects of environmental mismatch should be included in future work. Finally, giving the processor 
an inaccurate estimate of the jammers should be studied. This directly effects the results of the processor. 


\section{REFERENCES}

[1] A. Tolstoy, Matched Field Processing for Underwater Acoustics, World Scientific, 1993.

[2] A.B. Coppens, A.R. Frey, L.E. Kinsler, and J.V. Sanders, Fundamentals of Acoustics, Wiley, 1982.

[3] D.E. Dudgeon and D.H. Johnson, Array Signal Processing Concepts and Techniques, Prentice Hall, 1993.

[4] I.P. Kirsteins, Analysis of Reduced-Rank Interference Cancellation, Ph.D. Dissertation, University of Rhode Island, Kingston, R.I., 1991.

[5] I.P. Kirsteins, "A Reduced-Rank Generalized Likelihood Ratio Test, " NATO ASI Conference on Acoustic Signal Processing for Ocean Exploration, Madeira, Portugal, July 1992.

[6] E.J. Kelly, "an Adaptive Detection Algorithm," IEEE Trans. Aerospace and Electronic Systems, Vol. AES 23 No.4, pp. 115-127, Nov. 1986.

[7] S.L. Marple Jr., Digital Spectral Analysis with Application, Prentice Hall, 1987.

[8] K.R. Gabriel, "Least Squares Approximation of Matrices by Additive and Multiplicative Models," J.R. Statist. Soc. B., 40, No. 2, pp. 186-196, 1978. 
[9] H.B. Riley and J.A. Tague, "Matched Field Source Detection and Localization in High Noise Environments: A Novel Reduced-Rank Signal Processing Approach," Southeastern Symposium on System Theory, Tuscaloosa, Alabama, March 1993.

[10] G. Strang, Linear Algebra and Its Applications, Academic Press, Inc., New York, 1980.

[11] T.L. Marzetta, "A New Interpretation for Capon's Maximum Likelihood Method of Frequency-Wavenumber Spectral Estimation," IEEE Trans. on Acoustics, Speech, and Signal Processing, 31, pp. 445-449, April 1983.

[12] H.B. Riley, Matched-Field Source Detection and Localization in High Noise Environments: A Novel Reduced-Rank Signal Processing Approach, Ph.D. Dissertation, Ohio University, Athens, Ohio, 1993.

[13] I.P. Kirsteins and D.W. Tufts, "On the Probability Density of Signal-to-Noise Ratio in an Improved Adaptive Detector," ONR Technical Report, 1984.

[14] I.P. Kirsteins and D.W. Tufts, "On the Probability Density of Signal-to-Noise Ratio in an Improved Detector," Proc. IEEE International Conference on Acoustics, Speech and Signal Processing, pp. 572-575, 1985.

[15] R.J. Vaccaro, D.W. Tufts, and G.F. Boudreaux, "Advances in Principal Component Signal Processing," In: SVD and Signal Processing, F. Deprettere, ed., Elsevier Science Publishers, The Netherlands, pp. 115-146, 1988. 
[16] I.P. Kirsteins and D.W Tufts, "Rapidly Adaptive Nulling of Interference," Proc. GRETSI Conference, Juan-Les-Pins, France, June 1989.

[17] I.P. Kirsteins and D.W. Tufts, "Rapidly Adaptive Nulling of Interference," Proc. IEEE International Conference on Systems Engineering, Dayton, Ohio, pp. 269-272, Aug. 1989.

[18] B.D. Van Veen, and K.M. Buckley, "Beamforming: A Versatile Approach To Spatial Filtering," IEEE ASSP Magazine, pp. 4-24, April 1988.

[19] A.C. Radder, "On The Parabolic Equation Method for 'Water-Wave Propagation," J. Fluid Mech. 95, Part 1, pp. 159-176,1979.

[20] S.T. McDaniel, "Parabolic Approximation For Underwater Sound," J. Acoust. Soc. Am. 58 (6), pp. 1178-1185, December 1975.

[21] A. Tolstoy and Clay, Ocean Acoustics, McGraw-Hill, 1966.

[22] E.J. Kelly, "An Adaptive Detection Algorithm," IEEE Trans. Aerospace and Electronic Systems, Vol. AES-23, No.4, pp. 115-127, Nov.1986. 


\section{APPENDIX A}

\section{Files Used In Thesis Research}

function[tempzhl] $=$ twodsig $(\mathrm{H}, \mathrm{w}, \mathrm{zos}, \mathrm{zoe}$, rans, rane,z0,d,n,k,step $)$

$\%$ This $\mathrm{m}$-file will localize the source using the glrt signal processor.

$\%$

$\% \mathrm{H}$ is the depth distance to the bottom of the shallow water.

$\% \mathrm{w}$ is the frequency at which the wave is propagating.

$\%$ zos is the depth location of the source.

$\%$ zoe is the depth location of the jammer.

$\%$ rans is the range of the source.

$\%$ rane is the range of the jammer.

$\% \mathrm{n}$ is the number of fones (i.e. sensors).

$\% \mathrm{~d}=$ fone spacing;

$\% \mathrm{z} 0$ is the coordinate of the top fone.

$\% \mathrm{~L}$ is the coordinate of the bottom fone.

$\% \mathrm{z}$ is the vector which contains the fones.

$\%$ argm is the argument of the modal function.

$\%$ expons is the exponential term for the source.

$\%$ expone is the exponential term for the jammer.

$\%$

$\%$ usage: [tempzh1] $=\operatorname{mfp}(\mathrm{H}, \mathrm{w}, \mathrm{zos}, z o e$, rans, rane,z0,d,n,k,step $)$

jampow $=10$;

sigpow $=3.162277$;

wpow $=.1$

$\mathrm{jam}=1$;

$\mathrm{Nt}=1$;

$\mathrm{L}=(\mathrm{n}-1) * \mathrm{~d}+\mathrm{z} 0$

$\mathrm{z}=\mathrm{z} 0: \mathrm{d}: \mathrm{L} ; \quad \% 1 \mathrm{xn}$

$c=1500$;

$\mathrm{Num}=\left(\mathrm{H}^{*} \mathrm{w}\right) /(\mathrm{pi} * \mathrm{c})+0.5$;

$\mathrm{N}=$ floor(Num);

$\%$ Generate the vector of vertical wave numbers ( eigenvalues) and $\mathrm{Kn}$.

i

for $\mathrm{i}=1: \mathrm{N}$

$$
g a(i)=(i-0.5) *(p i / H) ; \% 1 x N
$$


$\mathrm{Kn}(\mathrm{i})=\operatorname{sqrt}\left((\mathrm{w} / \mathrm{c})^{\wedge} 2-\mathrm{ga}(\mathrm{i}) .^{\wedge} 2\right) ; \% 1 \times \mathrm{N}$

end

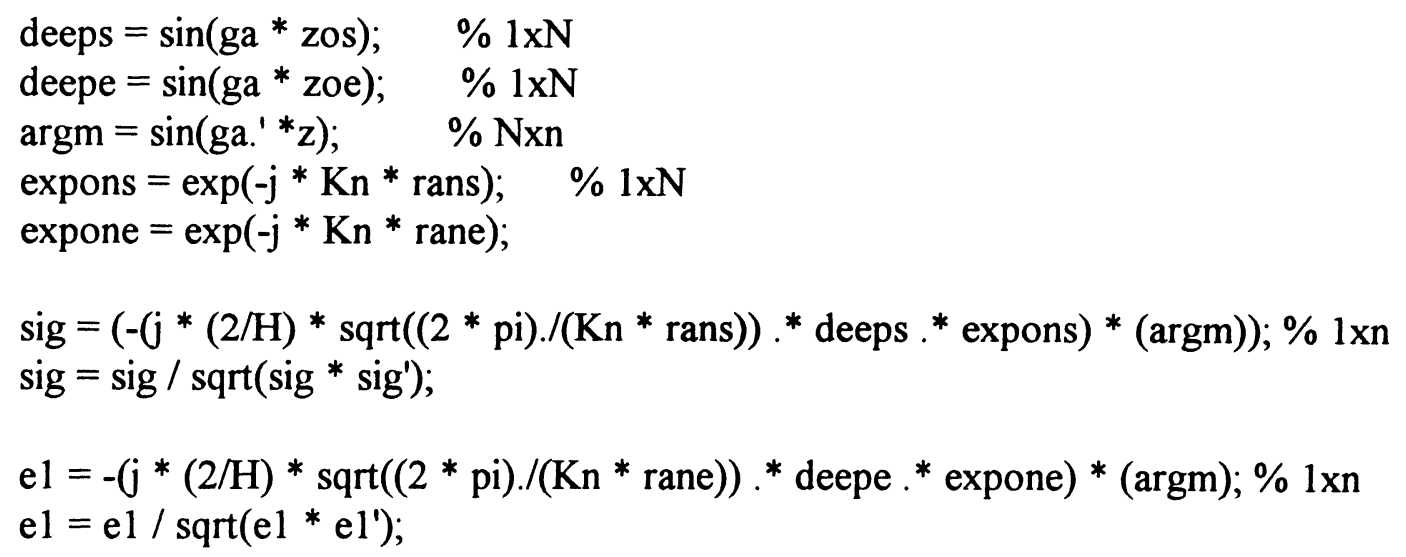

$\%$ compute the noise components.

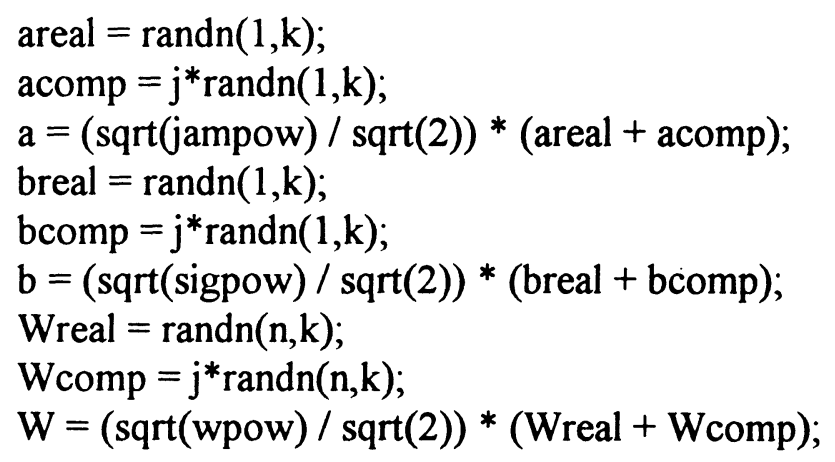

$\%$ Generate the data matrix $X$.

$$
\begin{aligned}
& X=\operatorname{sig} * b+e 1 * a+W ; \quad \% n x 1 * 1 x k+n x 1 * 1 x k+n x k=n x k \\
& \text { singno }=\operatorname{svd}(X)
\end{aligned}
$$

$\%$ Sweep through depth to locate signal.

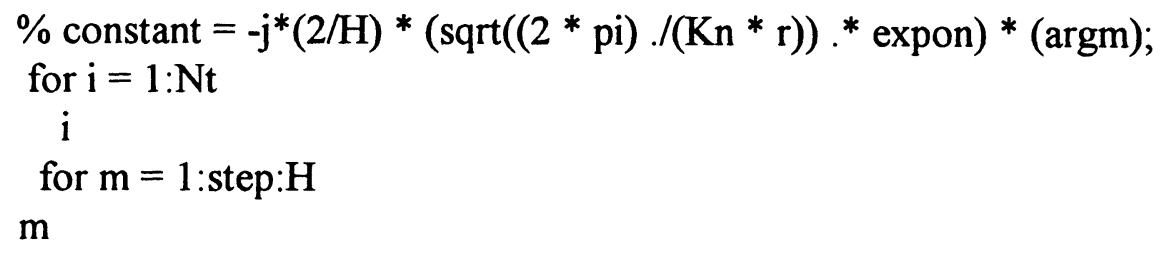




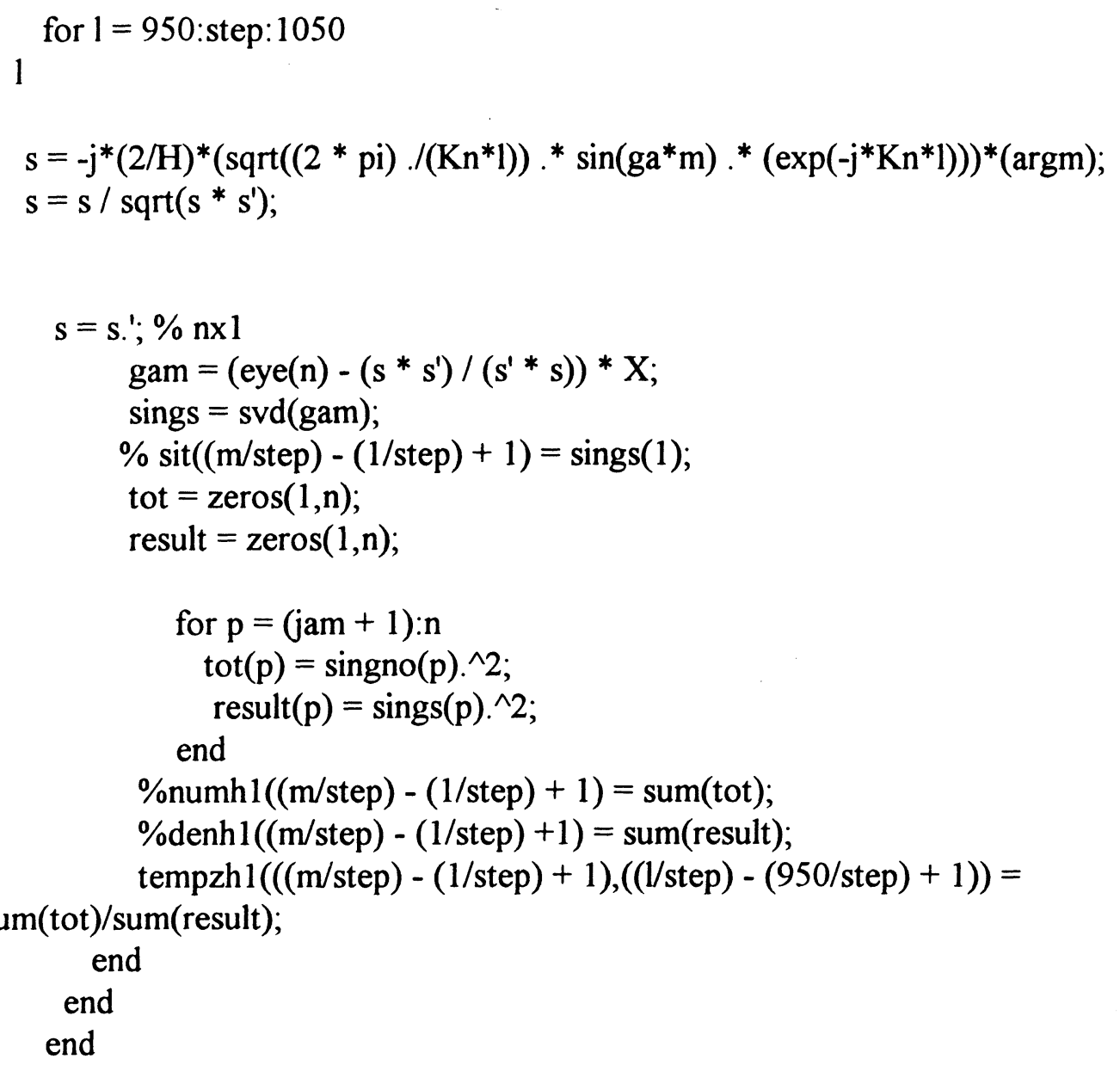

end

function[tempzhl] $=$ twodsig $(\mathrm{H}, \mathrm{w}, \mathrm{zos}, \mathrm{zoe}$, rans, rane,z0,d,n,k,step $)$

$\%$ This $\mathrm{m}$-file will localize the source using the glrt signal processing.

$\%$

$\% \mathrm{H}$ is the depth distance to the bottom of the shallow water.

$\% \mathrm{w}$ is the frequency at which the wave is propagating.

$\%$ zos is the depth location of the source.

$\%$ zoe is the depth location of the jammer.

$\%$ rans is the range of the source.

$\%$ rane is the range of the jammer.

$\% \mathrm{n}$ is the number of fones (i.e. sensors).

$\% \mathrm{~d}=$ fone spacing;

$\% \mathrm{z} 0$ is the coordinate of the top fone.

$\% \mathrm{~L}$ is the coordinate of the bottom fone. 
$\% \mathrm{z}$ is the vector which contains the fones.

$\%$ argm is the argument of the modal function.

$\%$ expons is the exponential term for the source.

$\%$ expone is the exponential term for the jammer.

$\%$

$\%$ usage: [tempzhl] $=\operatorname{mfp}(\mathrm{H}, \mathrm{w}, \mathrm{zos}, z o e$, rans, rane,z0,d,n,k,step $)$

jampow $=10$;

sigpow $=3.162277$;

wpow $=.1$;

jam $=1$

$\mathrm{Nt}=1$

$\mathrm{L}=(\mathrm{n}-1) * \mathrm{~d}+\mathrm{z} 0$;

$\mathrm{z}=\mathrm{z} 0: \mathrm{d}: \mathrm{L} ; \quad \% 1 \mathrm{xn}$

$c=1500$;

$\mathrm{Num}=\left(\mathrm{H}^{*} \mathrm{w}\right) /(\mathrm{pi} * \mathrm{c})+0.5$;

$\mathrm{N}=$ floor(Num);

$\%$ Generate the vector of vertical wave numbers ( eigenvalues) and $\mathrm{Kn}$.

for $\mathrm{i}=1: \mathrm{N}$

i

$\mathrm{ga}(\mathrm{i})=(\mathrm{i}-0.5) *(\mathrm{pi} / \mathrm{H}) ; \% 1 \mathrm{xN}$

$\mathrm{Kn}(\mathrm{i})=\operatorname{sqrt}\left((\mathrm{w} / \mathrm{c})^{\wedge} 2-\mathrm{ga}(\mathrm{i}) .^{\wedge} 2\right) ; \% 1 \mathrm{xN}$

end

deeps $=\sin (\mathrm{ga} * \mathrm{zos}) ; \quad \% 1 \mathrm{xN}$

deepe $=\sin (\mathrm{ga} *$ zoe $) ; \quad \% 1 \mathrm{xN}$

$\operatorname{argm}=\sin \left(g a .{ }^{*} z\right) ; \quad \% \mathrm{Nxn}$

expons $=\exp (-\mathrm{j} * \mathrm{Kn} *$ rans $) ; \quad \% 1 \mathrm{xN}$

expone $=\exp (-\mathrm{j} * \mathrm{Kn} *$ rane $)$;

$\operatorname{sig}=\left(-\left(\mathrm{j}^{*}(2 / \mathrm{H}) * \operatorname{sqrt}((2 * \mathrm{pi}) . /(\mathrm{Kn} *\right.\right.$ rans $)) .^{*}$ deeps.$^{*}$ expons $\left.) *(\operatorname{argm})\right) ; \% 1 \mathrm{xn}$

sig $=$ sig / sqrt (sig * sig');

e1 $=-\left(\mathrm{j} *(2 / \mathrm{H}) * \operatorname{sqrt}((2 * \mathrm{pi}) . /(\mathrm{Kn} *\right.$ rane $)) .{ }^{*}$ deepe.$*$ expone $) *(\operatorname{argm}) ; \% 1 \mathrm{xn}$

$\mathrm{e} 1=\mathrm{e} 1 / \operatorname{sqrt}\left(\mathrm{el} * \mathrm{el}{ }^{\prime}\right)$;

sig = sig.'; \% $\mathrm{nx} 1$

el $=\mathrm{e} 1$. ; $\% \mathrm{nx} 1$ 
$\%$ compute the noise components.

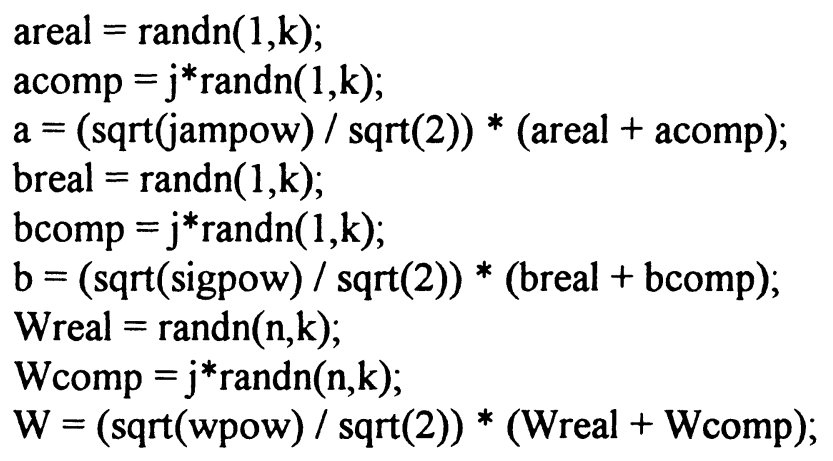

$\%$ Generate the data matrix X.

$$
\begin{aligned}
& X=\operatorname{sig} * b+e 1 * a+W ; \quad \% n x 1 * 1 x k+n x 1 * 1 x k+n x k=n x k \\
& \text { singno }=\operatorname{svd}(X)
\end{aligned}
$$

$\%$ Sweep through depth to locate signal.

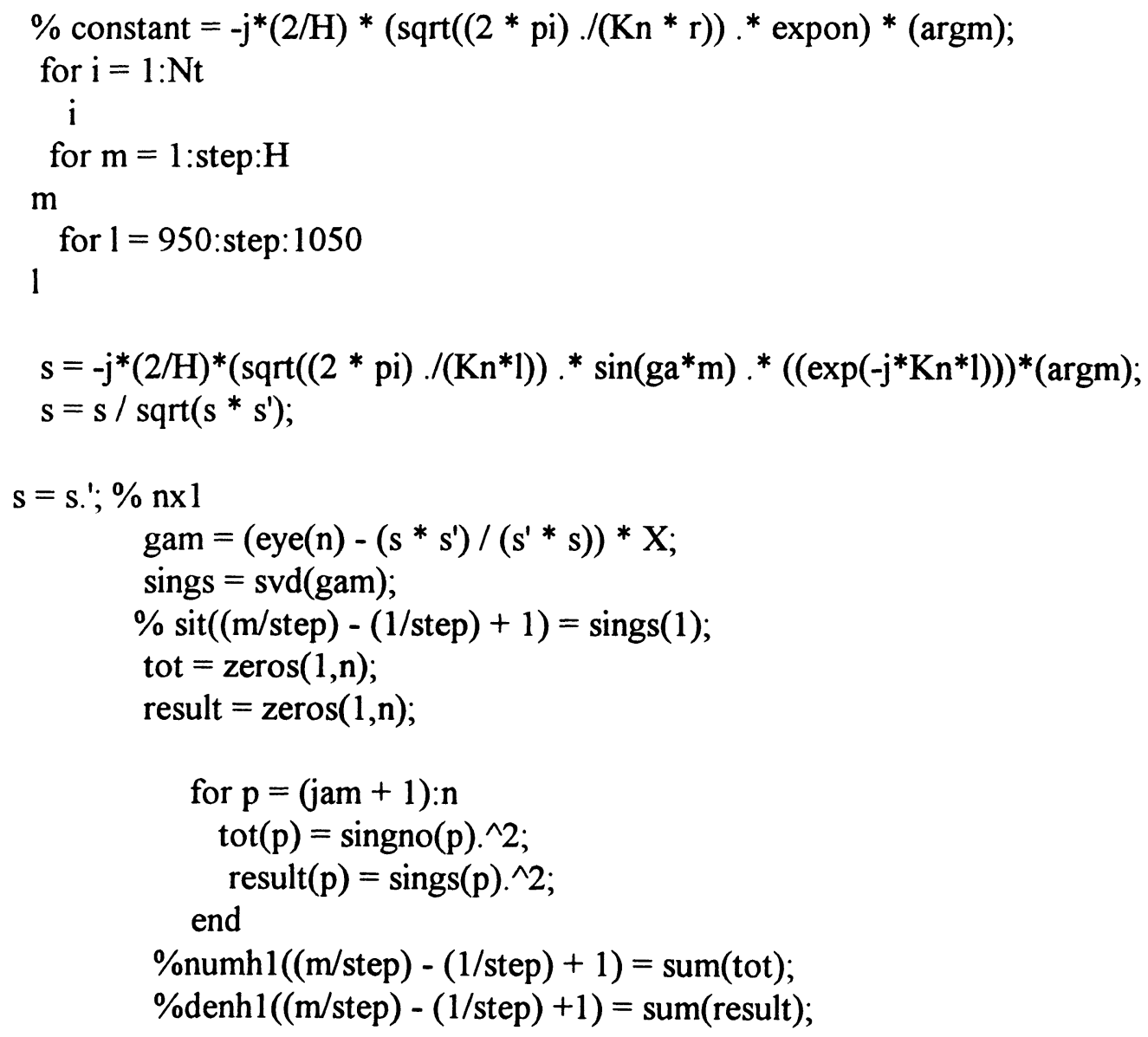


sum(tot)/sum(result);

tempzh1 $(((\mathrm{m} / \mathrm{step})-(1 / \mathrm{step})+1),((1 / \mathrm{step})-(950 /$ step $)+1))=$ end

end

end

end 